\title{
Design of a wavelength independent grating in the resonance domain
}

\author{
Tetsuya Hoshino, ${ }^{1, *}$ Saswatee Banerjee, ${ }_{1}^{1}$ Masahide Itoh, ${ }^{1}$ and Toyohiko \\ Yatagai ${ }^{1,2}$ \\ ${ }^{1}$ Institute of Applied Physics, University of Tsukuba, \\ 1-1-1 Tennoudai, Tsukuba 305-8577, Japan \\ ${ }^{2}$ Center for Optical Research and Education, Utsunomiya University, \\ 7-1-2 Yoto, Utsunomiya, Tochigi 321-8585, Japan \\ *Corresponding author: t-hoshino@optlab2.bk.tsukuba.ac.jp
}

\begin{abstract}
We propose using blazed gratings in the resonance domain with period larger than the wavelength for antireflection and polarization selection. The inherent problem in this region is wavelength dispersion, which is solved by analyzing the total reflectivity and electric field distribution. The positional relationship between the area of strong electric field, and the side and tip of the grating is crucial to the wavelength dispersion of total reflectivity. (c) 2008 Optical Society of America
\end{abstract}

OCIS codes: 050.0050, 050.1950, 050.1970, 230.1950, 230.3990.

\section{Introduction}

Fine subwavelength structures can be applied to optical components of flat panel displays or light emitting diode (LED) devices for lighting, and such structures have been discussed by many authors. The components of such structures are antireflective structures and polarization separation elements. [1]- [3] Some of the antireflective structures are designed using photonic crystals, [4] while others are constructed by a simple grating of a subwavelength period. [5] By applying these structures to the backlight of a liquid crystal display, or organic or inorganic LEDs, luminosity can be improved or their energy consumption can be reduced.

Various antireflective structures are used in light emitting elements. For example, antireflective photonic crystals are mainly used for inorganic LEDs [4]- [11] or organic LED(OLED) [12]- [16] . Some of the common gratings or microlenses are also used for LEDs or OLEDs as antireflective structures. [17]- [25] When light goes from a structure with low refractive index 
to one with high refractive index, the reflection can be reduced by the structure, making the structure useful for solar cell panels [26], [27] or the front panel of displays. [28]

For the use of antireflection, subwavelength structures were fabricated by Kanamori et al., [5], [29] and Kasugai et al. [30] . Moreover, when light travels from a structure with high refractive index to air with low refractive index, the extraction efficiency is improved by using a structure with a period as long as the wavelength for first-order diffraction efficiency. [31] All of the structures were formed by reactive ion etching. Since the groove width is subwavelength and is fine, the structures are difficult to produce.

On the other hand, an antireflective structure which has a period larger than $1 \mu \mathrm{m}$ can be fabricated by machining provided the aspect ratio is less than 2 or 3. [32] Antireflective structures can be designed precisely and fabricated easily by machining for gratings of this period. In this paper and the previous paper, [33] we propose a new form of optical control using a grating in the resonance domain with a period of more than the wavelength.

In this paper, we also propose polarization selection by this grating. We consider improving the efficiency of light use of the designed polarization for LCD applications. [1], [2] The penetration light of unnecessary polarization is scattered by gratings in one type of polarization selection, [3] while gratings reflect unnecessary polarization in another type.

Of these two types, the optical efficiency of the type that reflects unnecessary polarization can be improved easily. In this type, there is a system which reflects the incident light by a multilayer mirror [34] and a system which reflects the incident light of the Brewster angle by a stack of plates. [35], [36] There is also a system which reflects the light which obliquely enters the dielectric grating of a subwavelength period, [37]- [41] and a system which reflects light by a metal grating of subwavelength period. [42], [43] Thus, there are various systems of polarization selection. However, it is desirable to reflect unnecessary polarization with only one grating in a liquid crystal display, to simplify the process of producing the backlight and to reduce the thickness. We considered reflecting one of the two polarizations of the vertical incident light by a one-sheet grating made of one material. This facilitates the optical design as perpendicular incidence is possible. Moreover, a one-sheet grating is easier to make.

According to Mie theory and Rayleigh scattering theory, the scattering decreases rapidly with increasing wavelength [44], [45], when the particle size is less than the wavelength. With a grating which has a period of about half a wavelength, it is known that the reflected light looks blue. [46] On the other hand, a grating with a sufficiently long period has no coloring effect. For example, for a prismatic film which has a $50 \mu \mathrm{m}$ pitch prism array, its coloring effect is small and the film is used for almost all liquid crystal displays of high-class cellular phones. [13]

When the period is longer than several dozen times the wavelength, the reflectivity is not dependent on wavelength and when the period is shorter than wavelength the reflectivity 
of blue light is more than that of red light. The residual period ranges from 1 to several dozen times the wavelength, and in this domain total reflectivity fluctuates greatly with wavelength. [33] In this research, this domain is defined as the resonance domain.

Compared with a prism array with a long pitch, the resonance domain has various features: it has fewer moiré fringes caused by interference with pixels of the display; the grating depth is shallower for the same aspect ratio; and the diffraction effect can easily be taken into account in the calculation.

However, in this period, the wavelength dispersions of the transmissivity and the diffraction angle cause a problem. Conventionally, the goals of low reflectivity and low wavelength dispersion have not been realized, [47] and so applications are restricted to solar cell panels [26], [27] or for monochromatic light. [47], [48]

The limitation of the antireflective capability in the resonance domain has been examined, and its possibility as an optical element was exploited by resolving the problem of wavelength dispersion. [33] We previously described the wavelength dispersion of diffraction angle. [33] In this paper, we analyze the wavelength dispersion of total reflectivity and develop a method to suppress wavelength dispersion. First, the reflectivity of the grating in TE and TM modes is measured by experiment, and then compared with the reflectivity calculated by rigorous coupled wave analysis (RCWA). We also examine the limitation of the antireflective performance. Moreover, we analyze the electric field by nonstandard finite-difference time-domain (NS-FDTD) [49]- [53] calculation to clarify the relationship between the field distribution and reflectivity, and exploit ways to enhance transmissivity due to the grating, and to reduce the wavelength dispersion of reflectivity.

Our examination consists of three parts. The first is to devise a grating with a new function or to improve the function. The second is to check the function by experiment. The third is to explain the important optical characteristics of the grating by simple theory.

\section{Sample Preparation and Measurement}

\section{A. Grating for measurement}

Sample preparation and shape evaluation were performed as follows.

Two types of gratings were used for antireflection and polarization separation. One grating (Yuki Kougaku Co., Ltd.) was made by molding, and was used for reflectivity measurement of the surface relief of the grating shown in Fig. 1. It is made of PMMA (poly methyl methacrylate) and has a refractive index of 1.49. The grating profile is an isosceles triangle with the depth of $7 \mu \mathrm{m}$ and the period of $10 \mu \mathrm{m}$. The substrate thickness is $2 \mathrm{~mm}$. The average surface roughness, which is defined by the deviation from the central line in a direction parallel to the ridgeline, is $60 \mathrm{~nm}$.

Atomic force microscopy (AFM) and scanning electron microscopy (SEM) were used to 
measure the depth and period of the grating. SEM was used for measuring the depth and shape, and AFM was used for measuring the period and surface coarseness. Nanopics (Seiko Instruments Inc.) was used for AFM analysis, and TINY-SEM (Technex Co., Ltd.) for SEM analysis.

Next, the method of fabricating the grating for polarization separation is described. The grating was produced by transferring it from a metallic mold produced by machining. The mold was made by an ultra-precision micro-processor (NIC-200, NAGASE INTEGREX Co., Ltd.), having non-contact hydrostatic bearings.

The metallic mold was made from copper. As shown in Fig. 2, the metallic mold was a disk with a diameter of $15 \mathrm{~mm}$. Four kinds of gratings were curved on the metallic mold at intervals of $3 \mathrm{~mm}$. The periods of the gratings were 1.08, 1.8, 3 and $5 \mu \mathrm{m}$ from left to right in Fig. 2. The width of the area of each grating was $1 \mathrm{~mm}$.

The grating was transferred by UV curing from this metallic mold. UV curing resin was applied to the metallic mold, and a glass slide (Matsunami Glass Ind., Ltd.) was pressed on it. It was cured by being exposed to UV light, then the grating was removed from the mold.

The UV curing resin was SANRAD RC610-R (Sanyo Chemical Industries), and its refractive index after curing was 1.52 .

A UV mask aligner (M-2L type, MIKASA Co., Ltd.) was used for UV exposure. The lamp current of the super-high-pressure mercury lamp was set at $6.3 \mathrm{~A}$, lamp voltage at $40 \mathrm{~V}$, and exposure time at 35 seconds.

The aspect ratio of the transferred groove was 0.48 for any period of the gratings, and the average surface roughness of the grating was $18 \mathrm{~nm}$.

The substrate thickness of the grating was measured by a confocal microscope. The thickness was measured on the even portion near the groove where reflectivity was measured.

\section{B. Measurement of Total Reflectivity}

First, the reflectivity was measured using the above-mentioned grating with the period of $10 \mu \mathrm{m}$. The reflectivity of the relief surface of the grating was measured as shown in Fig. 1. The concave-convex surface is denoted by "relief" and the rear surface is denoted by "base". In order to suppress the reflection from the base, a dove prism was attached to the base of the grating by the UV curing resin as shown in Fig. 1. The attached prism looks like the tail of a standing pigeon. The grating is curved on a clear plastic board and has striped grooves. The longest side of the dove prism is parallel to the striped grooves.

The incident light is diffracted by the surface relief, enters the prism, is reflected several times and is diffused. The refractive index of the dove prism is 1.52. The light source was an Ar ion laser (Spectra-Physics Stabilite 2017), with a wavelength of $514.5 \mathrm{~nm}$.

The reflectivity was measured as follows, assuming the case where light enters the surface 
relief from air as shown in Fig. 1. Only the reflection from the surface relief is measured. Light was made to enter the grating and was reflected. The angle $\theta i$ between the normal line and incident light is $0^{\circ}$. An optical power meter (Thorlabs Inc.) was used to measure catoptric light, using photodiodes for its optical power meter sensors. The polarizer was placed between the light source and grating and the reflected light was measured separately for the TE and TM modes of incident light.

The power meter was used to measure the light around the grating at fixed intervals in the shape of a semicircle. The interval between measurement positions was made short enough to minimize errors. The sum of the reflectivity of each point was computed in consideration of the distance from the reflecting point of the surface and the area of the light-sensitive element of the power meter. The light of right reflection was disregarded as it could not be measured. The value of background light was subtracted from the measured value.

Next, the reflectivity of a grating with base was measured without the dove prism. Light was made to enter perpendicularly the surface relief of the grating from air. Strong 632.8 $\mathrm{nm}$ light from the He-Ne laser was used. The total reflectivity except right reflection was measured with the light in TE mode or TM mode by the power meter, and the ratio of TE to TM reflectivity was computed from this total reflectivity.

\section{Calculation of Total Reflectivity and Electric field}

\section{A. Method of Calculation of Total Reflectivity}

The total reflectivity of the grating was computed using RCWA and the electric field was computed using NS-FDTD algorithms, for a grating with an isosceles triangular profile. It was calculated for the case where the interface was only the surface relief, and the case where the interface was both the surface relief and the base. The incidence angle was $0^{\circ}$. It was assumed that the refractive index of the substrate was independent of wavelength. Unless noted otherwise, the refractive index of the substrate is 1.5 and the refractive index of air is 1. Wavelength, aspect ratio, and polarization were changed suitably. Case A was when incident light entered the relief structure of the grating from air, and the opposite case of light direction was case B. DiffractMOD ${ }^{T M} 1.5$ (RSoft Design Group, Ossining, NY, USA) was used to calculate reflectivity. DiffractMOD ${ }^{T M}$ is based on the RCWA method.

\section{B. Method of Calculation of Electric Field}

The NS-FDTD program developed at Tsukuba University was improved and used to calculate the electric field. This program was run under MathCAD ${ }^{T M} 2004$ (MathSoft Engineering and Education, Inc., Cambridge, USA). The minimum spatial step size $h$ was taken to be $\lambda / 10$, where $\lambda$ is wavelength of the light. $\lambda / h$ was set to be 10 . The number of calculation steps was 250 . The total calculation space employed here was $100 \lambda \times 32 \lambda$ along the $\mathrm{X}$ and 
$\mathrm{Y}$ axes, respectively. Incident light propagates along the $\mathrm{Y}$ axis. For perfect matching of the layer surrounding the calculation area, the Mur absorbing boundary condition was used. The incident light was a finite plane wave.

\section{Result of Experiment and Calculation}

\section{A. Triangular and Rectangular Grating}

At first, we discuss suitable shape of a grating for reducing reflection. Table 1 shows that the total reflectivity is dependent on the groove profile and refractive index. The groove profile is either a rectangular shape or isosceles triangular shape, the fill factor is 0.5 , the spatial period $\Lambda$ is $9.1 \lambda$, and $d / \Lambda$ is 1 . A parallel beam of light has the incidence angle $\theta i=0^{\circ}$, where $\theta i$ is incidence angle, while the incidence angle for diffusion light varies from 0 to $80^{\circ}$ and its intensity is uniform regardless of the angle. The refractive index of the groove of the grating is 1.5 or 3 . The table shows that a triangular grating has smaller reflectivity when the incident light is parallel light. Therefore, the groove of an isosceles triangle was adopted in this study.

\section{B. Comparison of Experiment and Calculation}

The results of experiment and calculation were compared so that we can verify that the calculation is valid. Table 2 shows the case where the interface is only the surface relief of the grating in case A. The grating used in the experiment had the period of $10 \mu \mathrm{m}$. Although the result in TM mode in which reflectivity was small did not agree with the calculation, the result in TE mode in which reflectivity was large agreed with the calculation. These results show that the required experimental accuracy in TM mode is higher than that in TE mode, as the reflection of TM mode is the smaller.

Next, in case A we examined the case where the interface of the grating was both the relief structure and the flat base of the grating. Since the influence of thickness $d s$ on this reflectivity was large, $d s$ should be determined for calculation. Thickness was measured using the confocal microscope, and reflectivity was calculated at intervals of $0.05 \mu \mathrm{m}$ in the range of observed $d s \pm 0.15 \mu \mathrm{m}$. The ratio of TE to TM at which the results of the experiment and calculation agreed well was searched for by adjusting the thickness. The thickness was changed in the range of the measured value $\pm 0.1 \mu \mathrm{m}$, and the reflection ratio of TE to TM was obtained from the result. The substrate thickness is shown in Table 3. In consideration of the variation of the thickness $d s$ of the substrate with the inclination of the thickness, the average reflectivity was calculated at three points which included two points whose thicknesses were $d s \pm 0.05 \mu \mathrm{m}$. The calculated ratio of the total reflectivity of TE to TM is shown in Table 4, and the thickness is shown in Table 3. The average deviation of the reflection for each grating was $13 \%$, so the experiment and calculation results were in 
reasonable agreement.

From the above results, two applications appear promising. One is antireflection and the other is polarization selection. The grating without base is for antireflection and the grating with base is for polarization selection.

These can be used for displays or lighting. However, the problem is wavelength dependence of reflectivity or polarization selectivity.

\section{C. Calculation of Total Reflectivity for Surface Relief}

Next, the total reflectivity was calculated as a design indicator of the antireflectivity. Figure 3 shows the dependence of computed reflectivity on wavelength on surface relief at normal incidence in case A. The vertical axis is total reflectivity and the horizontal axis is $\Lambda / \lambda$. In both TE mode and TM mode, $\Lambda / \lambda$ corresponding to a peak is near an integer value 1,2 , $3, \ldots$ periodically. Thus, there is PRPG (periodicity of total reflectivity against period of grating). The refractive index is 1.5 , and the aspect ratio varies from 0.5 to 2 . Although not shown here, the same PRPG was seen in case B. [33]

In the case of a rectangle of aspect ratio 2 , in the range of $n d / \lambda$ of 1 to 4 , reflectivity fluctuated with the period of 0.5 against $n d / \lambda$, where $n$ is refractive index of the grating. Its periodicity was not dependent on $\Lambda / \lambda$, but dependent on $d / \lambda$.

Next, in order to explore the mechanism of PRPG and the difference of TE and TM, the total reflectivity was calculated for different aspect ratios. As shown in Fig. 4, the incidence angle to the slope of the groove was considered. The result is shown in Figs. 5 to 8, with the angle $\theta$ on the horizontal axis. These figures show the relationship of total reflectivity and incidence angle $\theta$. The incidence angle $\theta$ varied with the aspect ratio, which was defined by $d / \Lambda$.

In Figs. 5 and $7, \Lambda / \lambda$ is 9.1 and refractive index varies from 1.5 to 3 . Moreover, $n$ of 1.2 is added to Fig. 7. Figures 5 and 7 show the refractive-index dependence of the reflectivity of incidence perpendicular to the slope of the grating, calculated for both TE and TM modes. Figure 5 is case A and Fig. 7 is case B.

In Figs. 6 and $8, \Lambda / \lambda$ is 9.1 or 18.2 and refractive index is 1.5 or 3 . Figures 6 and 8 show the refractive-index dependence of the reflectivity of incidence perpendicular to the slope of the grating, calculated for both TE and TM modes. Figure 6 is case A and Fig. 8 is case B.

In Fig. 7 and 8 , there is a peak near 45 or $67.5^{\circ}$ with a refractive index of 3 . The peak of the reflection for high refractive index is shifted to a small incident angle, when it is compared with the peak for low refractive index. Figure 9 is a schematic diagram of the reflection based on the ray optics near these peaks. 


\section{D. Calculation of Electric Field for Surface Relief}

The electric field was calculated in order to examine the causes of PRPG and that of the difference of total reflectivity by polarization in detail. The results are shown in Figs. 10 to 18. The deep color of an electric field indicates a large plus or minus value. The scale of the relative value is shown in Fig. 10(c). The grating is shown by the black dotted line. Incident light enters from the left end. Figure 10 shows the electric field of incident light and scattered light, and Figs. 11 to 18 show the electric field of scattered light.

In Fig. 10(c) the electric field inside the grating has a different interval from that outside. Regarding the direction of wave propagation, the number of waves in the grating is 9 , and that outside the grating is 7 . There are more waves in the grating than outside the grating, which may cause discrepancy of the stripe of the electric field. Moreover, there are three areas surrounding the peak in a grating parallel to the base. We call this area with strong electric field intensity simply "area".

In Fig. 11, the period is $2 \lambda$. The electric field parallel to the base has one area in the middle, and half at both ends. On the other hand, when the calculation was performed for a rectangular grating with the fill factor of 0.5 , although 4.5 phases were found in the direction of $\mathrm{Y}$ direction in the grating with the same period, the gratings did not have a clear number of phases in the $\mathrm{X}$ direction.

In Fig. 12, the period is $3 \lambda$. The electric field parallel to the base has two areas in the middle, and half at both ends. In Fig. 13, the period is $4 \lambda$. The electric field parallel to the base has three areas in the middle, and half at both ends. The number of phases is not clear in the grating with aspect ratio of 0.5 or 1 . On the other hand, the electric field of the rectangular grating has 9 areas along the $\mathrm{Y}$ axis, but is not clear along the $\mathrm{X}$ axis, as shown in Fig. 14.

Figure 15 shows the result of the calculation for TM mode. In TE mode of case A of a triangular grating, the number of areas is independent of the aspect ratio as shown before, and this independence applies to TM mode, also. Thus, the period plays an important role in the phase distribution.

TM mode has X phase and Y phase, whereas TE mode has only one phase in the plane. If the aspect ratio changes, although the number of phases does not change in $\mathrm{Y}$ phase, it changes in $\mathrm{X}$ phase. For example, for the period of $4 \lambda$ although the number of areas in a grating does not change in $\mathrm{Y}$ phase when the aspect ratio changes to 3 from 1, it increases from 6 to 9 in X phase. As we considered that Y phase vertical to the incident light had a large influence on PRPG, we employed Y phase in this study. For Y phase in TM mode, the electric field phase distribution was the same as in TE mode.

As the Mur absorbing boundary condition is used, it is difficult to assume an infinite area when performing the calculation for a system with an interface, such as an object with infinite 
$d s$. Therefore, we used the grating whose $d s$ is 0 for the object. The magnitude relation of the reflectivity was newly calculated by RCWA. The electric field was calculated for both case $\mathrm{B}$ and case $\mathrm{A}$. The result of calculating the phase distribution of the perpendicular incidence in TE mode in case B is shown in Fig. 16. In this figure, the period is 2 and $4 \lambda$ and the aspect ratio is 1 . There are two and four phases in the portion between the tips of the grating, respectively. As shown, since the phase distribution of the electric field of case B does not depend on the aspect ratio but is proportional to $\Lambda$, the electric field distribution is likely to be related to PRPG. Therefore, we examined the relation between reflectivity and phase distribution in detail.

The results are shown in Figs. 17 and 18. In Fig. 17 the aspect ratio of the grating is 1.

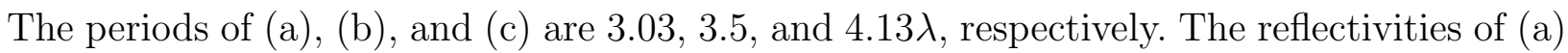
and (c) are low and that of (b) is high.

Figure 18 shows the result of calculating the phase distribution of the perpendicular incidence in TE mode in case B. The aspect ratio of the groove is 0.5. (a), (b), and (c) have periods of $2.84,3.03$, and $3.8 \lambda$, respectively. The reflectivities of (a) and (c) are high and that of (b) is low.

\section{E. Calculation of Total Reflectivity and Electric Field for Grating with Base}

Next, in order to acquire an indicator for designing the grating for polarization selection, the total reflectivity and the electric field phase of scattered light were calculated by varying $d s / \lambda$ as shown in Figs. 19 and 20.

The total reflectivity of the grating with substrate thickness was calculated as shown in Fig. 19. When $d s / \lambda$ changes from 0.55 to 0.65 , the reflectivity of TM mode changes significantly for the grating with aspect ratio of 0.5 . The ratio of TE to TM reflectivity also changes greatly.

In Fig. 20, (a), (b), and (c) are for $d s / \lambda$ of $0.55,0.65$, and 0.85 , respectively. The reflectivities of (a) and (c) are high and that of (b) is low. The reflectivity changes greatly with $d s / \lambda$, and (b) with low reflectivity shows a disordered distribution of electric field. There are six electric field areas on the base of the grating. In a grating, the electric field area is located in a line by turns, and forms a pyramid. The electric field near the base is drawn on the left in the schematic diagram.

Figure 21 shows reflectivity and polarization ratio against wavelength. In (a), the grating is made of a blazed groove and substrate, and is sandwiched between air layers. The aspect ratio is 1 . The refractive index of the grating is denoted by $n$ and is 1.5 . The polarizations are TE and TM and the incidence angle is $0^{\circ}$. When $\Lambda / \lambda$ is $9.1, d s / \lambda$ is 0.55 or 0.65 .

In (c) the grating is made of blazed groove, substrate 1 and substrate 2 from top to bottom, and is sandwiched between air layers, as shown in (b). The refractive index of substrate 1 
and the grating is 1.52 and that of substrate 2 is 1.575 . The thicknesses of substrate 1 and substrate 2 are denoted by $d 1$ and $d 2$, respectively. When $\Lambda / \lambda$ is $9.1, d 1 / \lambda$ is 3.64 and $d 2 / \lambda$ is 181.2 .

In (a), when $\Lambda / \lambda$ ranges from 2.4 to 11 , the reflectivity of TE is more than twice that of TM. On the other hand, in (c) when $\Lambda / \lambda$ ranges from 3.8 to 5 , the reflectivity of TE is more than twice that of TM.

Furthermore, in order to consider the existence of the Brewster angle effect of reflection at the base, the aspect ratio was changed. The calculated results are shown in Fig. 22. The horizontal axis is incidence angle to base $\theta_{b}$ which is calculated from the aspect ratio. This angle $\theta_{b}$ was calculated under the assumption that the incident light entered the base after being bent by the sloping side according to Snell's law. The polarizations are TE and TM, refractive indexes are 1.5 and 1.9 , and $d s / \lambda$ is 0.55 and 0.64 .

Table 5 shows the relationship of $\theta_{B r}$ and the angle of the local minimum in Fig. 22, where $\theta_{B r}$ is Brewster angle. The result of $d s / \lambda$ of 0.605 and 0.705 is added. If the local minimum is determined by Brewster angle, $\theta_{B r}$ may give the angle of the local minimum. There is a difference of a few degrees between $\theta_{B r}$ and the angle of the local minimum, which was found to be the error of Snell's law by another FDTD calculation. The calculation shows that the incidence angle is a few degrees less than that predicted by Snell's law.

\section{Discussion}

\section{A. The Periodicity of Fluctuation of Reflection due to Wavelength Change}

The triangular grating has less reflectivity than the rectangular grating. Therefore, the isosceles triangle is mainly discussed in this section. The factors influencing reflectivity were considered. Wavelength dependence to total reflectivity seems to be caused not only by the period, but also by the depth. However, PRPG is caused only by the period. Moreover, PRPG seems to be specific for a triangular grating, because the rectangular grating does not have such a periodicity and its electric field distribution is different from that of a triangular grating.

When the wavelength is changed, the total reflectivity changes periodically and has a local maximum for $\Lambda / \lambda=1,2,3, \ldots$ as shown in Fig. 3. This periodicity is independent of polarization and aspect ratio. As shown in Fig. 3, TE and TM modes have the same reflectivity wavelength dependence. However, the reflectivity of TE mode is different from that of TM mode by one order of magnitude.

\section{B. Effect of Period and Aspect Ratio on Total Reflectivity}

There are two types of triangular grating. One is grating with base and the other is one without base. We think the grating without base at first. 
Since reflectivity is generally different by $\mathrm{S}=\mathrm{TM}$ polarization and $\mathrm{P}=\mathrm{TE}$ polarization in the case of oblique incidence to a plane, the difference of this reflectivity can be explained. We consider the side of the grating as the incident plane for $\mathrm{S}$ or $\mathrm{P}$. The light vertically enters the stripe of the grating for TE and TM modes. Generally polarization S has less reflectivity than polarization P. The above consideration shows that TM mode has less reflectivity than TE mode.

Considering Brewster angle $\theta_{B r}$, the incidence angle to a slope was changed by changing the aspect ratio. The results are shown in Fig. 5 for case A and in Fig. 7 for case B.

According to Snell's law, in Fig. 4, when the refractive index is $1.5, \theta_{B r}$ is $56^{\circ}$, and when it is $2, \theta_{B r}$ is $63^{\circ}$. The total reflectivity of $\mathrm{S}$ polarization has a local minimum at the Brewster angle. Although TM mode is equivalent to $\mathrm{S}$ polarization, the local minimum in TM mode at these angles is not seen. Therefore, the Brewster angle cannot clearly explain the difference of TE and TM modes.

On the other hand, in Fig. 7, when the refractive index is $1.2, \theta_{B r}$ is $40^{\circ}$, and when the refractive index is $1.5, \theta_{B r}$ is $34^{\circ}$. A small local minimum is seen at these angles in TM mode, and the Brewster angle can explain this local minimum. Although physical optics could explain the difference of reflectivity in TE and TM modes in case B from these results, in case $\mathrm{A}$, this difference cannot be explained simply by physical optics.

The reflection in case $\mathrm{A}$ for incidence angle $\theta$ is shown in Fig. 6. The total reflectivity of the grating with $\Lambda / \lambda=9.1$ is higher than that of the grating with $\Lambda / \lambda=18.2$. This relationship implies that the reflection of the tip of the grating is much higher than that of the sloping face of the grating, because as $\Lambda / \lambda$ becomes larger, the contribution of the tip to the reflection becomes smaller.

The reflection in case B is shown in Fig. 8. The total reflectivity of the grating with $\Lambda / \lambda$ $=9.1$ is not necessarily higher than that of the grating with $\Lambda / \lambda=18.2$. This relationship implies that the reflection of the tip of the grating is not higher than that of the sloping face of the grating. In Fig. 8, reflectivity has a local maximum when the inclination of the slope of the grating is changed.

Figure 9 shows the case where the light path is symmetrical and catoptric light returns in the direction opposite to the incident light. When incident light enters for the i-th time on the sloping face of the grating and is reflected on the slope, the incidence angle is set to $\alpha_{i}$. The angle $\alpha_{1}$ of (b) is $45^{\circ}$, and the angle $\alpha_{1}$ of (c) is $67.5^{\circ}$.

When the incidence angle is set to $\alpha_{1}$ and the apex angle is set to $2 \theta_{a}, \alpha_{1}=90^{\circ}-\theta_{a}$ and $\alpha_{1}+\alpha_{2}=2 \theta_{a}$ will hold true. When $\alpha_{1}$ is $40^{\circ}$ or less, it is smaller than the critical angle, and when $\alpha_{1}$ is about $45^{\circ}$, both $\alpha_{1}$ and $\alpha_{2}$ are more than the critical angle. For this reason, catoptric light increases as $\alpha_{1}$ increases. Furthermore, if $\alpha_{1}$ becomes larger than $50^{\circ}$, since $\alpha_{2}$ will become less than the critical angle, reflectivity decreases. The local maximum occurs 
under such change of reflectivity. Moreover, $\alpha_{1}$ becomes $67.5^{\circ}$ when the number of times of reflection is 4 . The same change of reflectivity occurs around the angle of $67.5^{\circ}$.

\section{C. Analysis of Electric Field for Surface Relief}

The above reflectivity shows both case A and B are useful for antireflection.

The reflectivity is large at the tip of the grating in case $\mathrm{A}$, and at the side of the grating in case B. We studied this difference of case A and case B using the fact of the periodicity of the total reflectivity by analyzing the electric field described below. Since the periodicity of total reflectivity against wavelength is dependent not on the depth of grating but on the period, we examined the electric field distribution in the direction parallel to the base of the triangle of the groove.

We considered that the width of the triangle of the grating had some effect on the reflection and so calculated the electric field. The results are shown in Figs. 10 to 18 for various gratings with different widths. In every case, the number of areas of the electric field along the base of the triangle changes in proportion to the width of it. In Fig. 15, the period of the grating is $2 \lambda$ or $4 \lambda$. Note the area of the electric field along the base of the groove indicated by the triangle. In the case of $2 \lambda$, one area in the center and the half area at two ends make two areas. On the other hand, in the case of $4 \lambda$, three areas in the center and the half area at two ends make four areas. In Fig. 13, the aspect ratio is $0.5,1,1.5$ or 3 , and although the aspect ratio differs, every grating has four areas along the base.

Thus, the number of phase areas along the rear surface of the grating increases as the period of the grating increases. The aspect ratio does not affect the number of phase areas along the rear surface of the grating. The results were the same for both TM mode as well as TE mode.

These results show that the number of areas along a base is proportional to a period. When the antinode of the electric field is on the edge, the reflectivity may rise, so we calculated the electric field of the wavelength which has high or low reflections. Since the influence of the tip part of a triangular grating on reflection might be large in case A, the relation between the electric field distribution of the tip part and reflectivity was examined for PRPG.

In Fig. 17, the electric field of the tip part is weak in (a) and (c) with small reflectivity, while it is strong in (b) with large reflectivity. We consider that the cause of PRPG is a rapid increase of reflection in the place where the electric field of the triangular end becomes a loop from the node of a wave. The difference between TE and TM modes is likely influenced by the reflection at the tip part more than that at the slope.

On the other hand, since the influence of the slope on reflection is large in case B, we examined the influence of the slope on reflective periodicity. The positional relationship between the area of the electric field and the side of the grating was checked. The electric 
field was examined for a grating with aspect ratio of 0.5 in which reflectivity has simple periodicity, which leads to the regular fluctuation curve of the total reflectivity in Fig. 3. In Fig. 18, the overlap of the sloping side of the grating and electric field is large in (a) and (c) with large reflectivity. However, the overlap is small in (b) with small reflectivity. This positional relationship between the sloping face of the grating and area of electric field is crucial for the reflectivity. Moreover, the reflectivity on the slope is much larger than that on the tip as shown in 5.B. Therefore, the difference between TE and TM modes may be caused by reflection on the slope because a slope has different transmissivity for different polarization. The positional relationship between the tip part or side of the grating and the area of the electric field determines the fluctuation of total reflectivity against wavelength. Thus there is a kind of interference between light and the tip part or the side in the direction perpendicular to the incident light.

\section{D. Grating with Different Substrate Thickness with Base in Case A}

Next, we think the grating with base. In case A, since the reflectivity of a grating with base increases greatly compared with a grating without it, the base of the grating is considered to largely influence the reflectivity.

In Fig. 19, the change of reflectivity as a function of substrate thickness was investigated in case A. When $d s / \lambda$ changes to $0.55,0.65$, or 0.85 , the reflectivity becomes high, low, and high, respectively.

These values are assigned to $d s / \lambda$ of (a), (b), and (c) in Fig. 20. The reflectivities of (a), (b), and (c) are high, low or high in this order. When the electric fields are investigated for (b), it turns out that the base is in the boundary of the electric field areas. We can say that (b) has low reflectivity because the base is in the node of an electric field. Thus there is a kind of interference in the direction parallel to the incident light, like thin film interference.

The above consideration shows that the grating with base can be used as polarization selection.

\section{E. Grating with Different Aspect Ratio with Base in Case A}

In Fig. 22, we examined the change of reflectivity as a function of the aspect ratio of the grating with base in case A. Although reflectivity in TE mode decreases monotonically with increase in aspect ratio, reflectivity in TM mode has a local minimum.

The total reflectivity against $d / \Lambda$ has the local minimum at the angle of $30^{\circ}$ when the refractive index is 1.5 , and at $27^{\circ}$ for the grating with refractive index of 1.9 . Thus, the angle of local minimum becomes smaller as refractive index increases.

Physical optics can explain this change by the concept that obliquely incident light is reflected at the base of a grating. That is, in TM mode, reflectivity may fall near the Brewster 
angle. Here, light is assumed to bend on the sloping face of the grating according to Snell's law and to enter the base. Since the angle at the local minimum becomes lower as the refractive index becomes higher in Fig. 22, this local minimum is explained by the Brewster angle.

\section{F. Design for Low Reflectivity and High Polarization Ratio}

We propose a method to reduce reflection as follows. From Figs. 5 and 8, when the number of interfaces is one and the aspect ratio is raised, reflectivity falls to some limiting value. As the reflectivity decreases in proportion to the angle, the lowest total reflectivity exists at the angle of $90^{\circ}$. The limitation is imposed by this total reflectivity.

When the grating has a base, it is possible to lower the total reflectivity of the TM mode. From Fig. 22, if polarization is TM mode when the grating has the base in case A, there is an aspect ratio with small reflectivity. Moreover, as the grating has the base in case A, the base can be arranged on the boundary of the area of the electric field in order to reduce the reflectivity. Moreover, in case A, it is effective to allow the local minimum of the intensity of electric field to come to both ends of a groove for lowering reflectivity.

Next, we consider why a high aspect ratio gives low reflectivity in order to clarify the mechanism of reflection of the triangular grating. The FDTD results of Fig. 13 (c) and (d) show that the wave front runs into the side of the grating at each line of electric field. As the aspect ratio becomes higher, the length of the side becomes longer, and so the number of points where light penetrates will increase. On the other hand, since the sides of the grating become steep inclines, it is hard for the light to penetrate the side of the grating, because generally, as the incidence angle to a plane becomes larger, the reflectivity becomes higher. These results show that the former effect dominates the latter.

Next, we describe a method to enhance polarization selectivity. As shown in Figs. 19 and 21 , the polarization ratio changed sharply with wavelength, and also becomes larger with higher aspect ratio.

In order to use this element for polarization separation of white light, the polarization ratio must be large in a certain wavelength range. The figures show that there is a domain of $\Lambda / \lambda$ where this can be used, even if the wavelength doubles.

As shown in Fig. 22, in TM mode, an incidence angle to the slope exists at which reflectivity becomes a local minimum. At this time, $\theta_{b}$ is $30^{\circ}$ and the aspect ratio is set to 0.9 when the refractive index is 1.5. As shown in Fig. 21(a), the polarization ratio of 20 is obtained near this incidence angle. 


\section{G. Design to Suppress Wavelength Dependence of the Total Reflectivity}

We studied the factors which influenced the wavelength dependence, examining the positional relation between the area of the electric field in the $\mathrm{X}$ and $\mathrm{Y}$ directions, and each side of a groove in Figs. 17, 18 and 20.

As shown in Figs. 21(a) and 22, reflectivity changes greatly with substrate thickness. Unlike a rectangular groove, for a blazed-grating groove, this change is not so periodic.

The fluctuation of reflectivity with wavelength arises not only in the direction perpendicular to the base but also in the direction parallel to the base. When the end of the groove of Fig. 20 reaches the point of minimum intensity of electric field, its reflection is small.

On the other hand, since the size of the area of electric field changes in Fig. 21 when the wavelength changes, the positional relation between the area and side changes and so reflectivity changes. The factor which influences the change in reflectivity is determined by the direction of incidence, and grating profile, as the positional relationship is influenced by these factors.

As described in section 5.C, the positional relationship between the area of electric field and the side or tip of the grating is crucial to the reflectivity. In the triangular groove of case A, since the reflectivity of both ends of a triangular groove is large, when the area of electric field comes to the tip of the grating, the reflectivity becomes high. Then, the change of the positional relationship is periodic to $\Lambda / \lambda$, as the size of the area is changed by $\Lambda / \lambda$. For this reason, peaks of $\Lambda / \lambda$ are near $1,2,3, \ldots$ Although the triangular groove of case $\mathrm{B}$ has large reflectivity in the sloping side of the groove, it has almost the same periodicity as the groove of case A.

From the above examination, to reduce the wavelength dependence of reflectivity in the resonance domain of a grating, the following designs are necessary.

The first is a method of enlarging a period which makes the reflectivity to the human eye constant irrespective of changes of wavelength in the visible light range, as shown below. Although the color sensors of the human eye are highly sensitive to the ratio of the three primary colors, their wavelength resolution is not high. Even if the wavelength changes in the wavelength range of the color sensor of red or blue, the color does not change if the ratio does not change.

For simplicity, let the wavelengths of red, green and blue be $\lambda_{R}, \lambda_{G}$ and $\lambda_{B}$, respectively. Let the ratio of $\lambda_{R}, \lambda_{G}$ and $\lambda_{B}$ be 8,7 and 6 , as we can use 620,520 and $460 \mathrm{~nm}$ for the three primary colors of the sensors of the human eye. The sensor of the human eye to red has a wider range of sensitivity than the difference of $\lambda_{R}$ and $\lambda_{G}$. The sensors for $\lambda_{R}, \lambda_{G}$ and $\lambda_{B}$ have similar ranges of sensitivity. The period of the grating is set to $7 \lambda_{G}$. The numbers of areas of the electric field of $\lambda_{R}, \lambda_{G}$ and $\lambda_{B}$ will be 6,7 and 8 as the number of areas is proportional to $\Lambda / \lambda$. Under the above conditions, the reflectivities of $\lambda_{R}, \lambda_{G}$ and $\lambda_{B}$ are 
almost the same, because the positional relationship of the electric field and the side of the grating are almost the same for each color. For example, when the area of electric field of $\lambda_{R}$ is on the edge of the grating, those of $\lambda_{G}$ and $\lambda_{B}$ are also on the edge. In this case, total reflectivity has one cycle of fluctuation, when the color changes from red to green, or from green to blue.

When the wavelength changes a little, the change of ratio in the three primary colors can be suppressed also for the period of grating larger than $7 \lambda_{G}$, because the area passes through the side of a groove repeatedly in the wavelength range of red or blue, and so the ratio of the colors does not change. When the wavelength changes from red to green, it is desirable for the area of an electric field to straddle the side of a groove at least once, which causes more than one cycle of fluctuation. Thus, if the period of the grating is more than $7 \lambda_{G}$, the ratio of the three primary colors can be constant when the color changes.

The second method is to vary the period and depth and to reduce wavelength dependence. If the grating is not periodic, the relationship between the area of electric field, and the surface relief and the base changes groove by groove. Thus, multiple positional relations between a side and an electric field area are created due to variations of the grooves. When the wavelength changes, the positional relation as a whole between grooves and electric field will not change for such grooves. Regarding the magnitude of the modulation of period and the depth of the groove, it is desirable to set it larger than the area of the electric field.

\section{Conclusion}

In the resonance domain of a grating, we proposed a new antireflective structure and a new polarizer which have simple structures, high transmissivity and small wavelength dependence.

The antireflective structure consists of only a relief. When the aspect ratio of the triangular grating is 0.7 and $\Lambda / \lambda$ is 19 , the total reflectivity reaches $0.4 \%$ as the average of the polarizations for both experiment and calculation. Furthermore, a technique for reducing reflectivity for a triangular grating was proposed. In the case of perpendicular incidence, the greater the aspect ratio is and the longer the period is, the smaller the reflectivity becomes. When the aspect ratio is 2 , the period is $18.2 \lambda$ and the refractive index is 1.5 , the total reflectivity becomes $0.1 \%$ for TE mode.

The polarizer structure consists of a relief and a substrate. A local maximum of the polarization ratio exists as the aspect ratio changes, and the polarization ratio fluctuates with changes in substrate thickness. When the aspect ratio is 0.9 and the substrate thickness is changed so that the polarization ratio is maximized, the ratio of the reflectivity of TE to TM reaches 20 and the transmissivity in TM mode is $99 \%$.

Furthermore, we calculated the electric field of the grating by NS-FDTD to find way to reduce the wavelength dependence of the total reflectivity. The areas of strong regular 
electric field are formed like the blocks of a pyramid in the groove of the grating, and the reflectivity increases when the area, and the side or the base are in the same location. As a result, the wavelength dependence of total reflectivity is governed by two factors, the period of the grating and the thickness of the substrate, because when the wavelength changes, the positional relationship changes and then the reflectivity fluctuates.

It is concluded that the design criteria for reducing the wavelength dependence of total reflectivity are to modify or resize the size of the period and the depth of the grating so that the side of the grating has multiple positional relations to the electric field in the range of color sensors of the human eye.

Thus, in the resonance domain, we desinged the optical elements with low total reflectivity and high polarization ratio and we also devised the methods to reduce wavelength dependence of them.

\section{Acknowledgments}

The authors thank Mr. J. Sugisaka, Dr. T. Suemasu and Mr. S. Sugawara for their contributions to the FDTD calculation and evaluation of the shape of the gratings. The authors also thank Dr. J.B. Cole for his original program of NS-FDTD.

\section{References}

1. K.-W. Chien and H.-P. D. Shieh, "Design and fabrication of an integrated polarized light guide for liquid-crystal-display illumination," Appl. Opt. 43(9), 1830-1834 (2004).

2. X. Yang, Y. Yan, and G. Jin, "Polarized light-guide plate for liquid crystal display," Opt. Express 13(21), 8349-8356 (2005).

3. J.-H. Park, C.-J. Yu, J. Kim, S.-Y. Chung, and S.-D. Lee, "Concept of a liquid-crystal polarization beamsplitter based on binary phase gratings," Appl. Phys. Lett. 83(10), 1918-1920 (2003).

4. S. Fan, P. R. Villeneuve, J. Joannopoulos, and E. Shubert, "High Extraction Efficiency of Spontaneous Emission from Slabs of Photonic Crystals," Phys. Rev. Lett. 78(17), 3294-3297 (1997).

5. Y. Kanamori, M.Sasaki, and K. Hane, "Broadband antireflection gratings fabricated upon silicon substrates," Opt. Lett. 24(20), 1422-1424 (1999).

6. H. Ichikawa and T. Baba, "Efficiency enhancement in a light-emitting diode with a twodimensional surface grating photonic crystal," Appl. Phys. Lett. 84(4), 457-459 (2003).

7. Y.-G. Ju, G. Almuneau, T.-H. Kim, and B.-W. Lee, "Numerical Analysis of High-Index Nano-Composite Encapsulant for Light-Emitting Diodes," Jpn. J. Appl. Phys. 45(4A), 2546-2549 (2006). 
8. A. Adawi, R. Kullock, J. Turner, C. Vasilev, D. Lidzey, A. Tahraoui, P. Fry, D. Gibson, E. Smith, C. Foden, M. Roberts, F. Qureshi, and N. Athanassopoulou, "Improving the light extraction efficiency of polymeric light emitting diodes using two-dimensional photonic crystals," Organic Electronics 7, 222-228 (2006).

9. H. K. Cho, J. Jang, J.-H. Choi, J. Choi, J. Kim, J. S. Lee, B. Lee, Y. H. Choe, K.-D. Lee, S. H. Kim, K. Lee, S.-K. Kim, and Y.-H. Lee, "Light extraction enhancement from nanoimprinted photonic crystal GaN-based blue lightemitting diodes," Opt. Express 14(19), 8654-8660 (2006).

10. J. Shakya, K. H. Kim, J. Y. Lin, and H. X. Jiang, "Enhanced light extraction in IIInitride ultraviolet photonic crystal light-emitting diodes," Appl. Phys. Lett. 85(1), 142 144 (2004).

11. H.-Y. Ryu, J.-K. Hwang, D.-S. Song, I.-Y. Han, , Y.-H. Lee, and D.-H. Jang, "Effect of nonradiative recombination on light emitting of two-dimensional photonic crystal slab structures," Appl. Phys. Lett. 78(9), 1174-1176 (2001).

12. Y.-C. Kim, S.-H. Cho, and Y.-W. Song, "Planarized SiNx/spin-on-glass photonic crystal organic light-emitting diodes," Appl. Phys. Lett. 89(173502), 1-3 (2006).

13. B. C. Krummacher, M. K. Mathai, V. Choong, S. A. Choulis, , F. So, and A. Winnacker, "General method to evaluate substrate surface modification techniques for light extraction enhancement of organic light emitting diodes," J. Appl. Phys. 100(054702), 1-6 (2006).

14. Y. Do, Y. Kim, Y.-W. Song, C.-O. Cho, H. Jeon, Y.-J. Lee, S.-H. Kim, and Y.-H. Lee, "Enhanced Light Extraction from Organic Light-Emitting Diodes with 2D SiO2/SiNx Photonic Crystals," Advanced Materials 15(14), 1214-1218 (2003).

15. Y. R. Do, Y.-C. Kim, Y.-W. Song, and Y.-H. Lee, "Enhanced light extraction efficiency from organic light emitting diodes by insertion of a two-dimensional photonic crystal structure," J. Appl. Phys. 96(12), 7629-7636 (2004).

16. M. Fujita, K. Ishihara, T. Ueno, T. Asano, S. Noda, H. Ohata, T. Tsuji, H. Nakada, and N. Shimoji, "Optical and Electrical Characteristics of Organic Light-Emitting Diodes with Two-Dimensional Photonic Crystals in Organic/Electrode Layers," Jpn. J. Appl. Phys. 44(6A), 3669-3677 (2005).

17. Y. Kanamori, K. Kobayashi, H. Yugami, and K. Hane, "Subwavelength Antireflection Gratings for GaSb in Visible and Near-Infrared," J. Appl. Phys. Part 1 42, 4020-4023 (2003).

18. K. Asakawa and T. Hiraoka, "Nanopatterning with Microdomains of Block Copolymers using Reactive-Ion Etching Selectivity," Jpn. J. Appl. Phys. 41, 6112-6118 (2002).

19. T. Nakamura, N. Tsutsumi, N. Juni, and H. Fujii, "Thin-film waveguiding mode light extraction in organic electroluminescent," J. Appl. Phys. 054505, 1-5 (2005). 
20. A. Kitamura, S. Naka, H. Okada, and H. Onnagawa, "Improved Light Outcoupling in Organic Electroluminescent Devices with Random Dots," Jpn. J. Appl. Phys. 45(1B), 613-616 (2005).

21. H. Kikuta, S. Hino, A. Maruyama, and A. Mizutani, "Estimation method for the light extraction efficiency of light-emitting elements with a rigorous grating diffraction theory," J. Opt. Soc. Am. A 23(5), 1207-1213 (2006).

22. H. W. Choia and S. J. Chua, "Honeycomb GaN micro-light-emitting diodes," J. Vac. Sci. Technol. B 24(2), 1071-1023 (2006).

23. M. Khizar, Z. Y. Fan, K. H. Kim, J. Y. Lin, and H. X. Jiang, "Nitride deep-ultraviolet light-emitting diodes with microlens array," Appl. Phys. Lett. 86(173504), 1-3 (2005).

24. N. C. Dasa, "Increase in midwave infrared light emitting diode light output due to substrate thinning and texturing," Appl. Phys. Lett. 90(011111), 1-3 (2007).

25. S. Kiyohara, M. Fujiwara, F. Matsubayashi, and K. Mori, "Organic Light-Emitting Microdevices Fabricated by Nanoimprinting Technology Using Diamond Molds," Jpn. J. Appl. Phys. 44(6A), 3686-3690 (2005).

26. S. Hava and M. Auslender, "Design and analysis of low-reflection grating microstructures for a solar energy absorber," Solar Energy Materials and Solar Cells 61, 143-151 (2000).

27. A. Parretta, A. Sarno, P. Tortora, H. Yakubu, P. M. J. Zhao, and A. Wang, "Angledependent reflectance measurements on photovoltaic materials and solar cells," Opt. Commun. 172, 139-151 (1999).

28. A. M. Nuijs and J. J. L. Horikx, "Diffraction and scattering at antiglare structures for display devices," Appl. Opt. 33(18), 4058-4068 (1994).

29. Y. Kanamori, H. Kikuta, and K. Hane, "Broadband Antireflection gratings for Glass Substrates Fabricated Fast Atom Beam Etching," Jpn. J. Appl. Phys. 39(7B), L735L737 (2000).

30. H. Kasugai, Y. Miyake, A. Honshio, S. Mishima, T. Kawashima, K. Iida, M. Iwaya, S. Kamiyama, H. Amano, I. Akasaki, H. Kinoshita, and H. Shiomi, "High-Efficiency Nitride-Based Light-Emitting Diodes with Moth-Eye Structure," Jpn. J. Appl. Phys. 44, 7414-7417 (2005).

31. A. Fujimoto and K. Asakawa, "Nano-structured surface fabrication for higher luminescent LED by self-assembled block copolymer," in 2005 International Microprocesses and Nanotechnology Conference, pp. 76-77 (Tokyo, Japan, 2005).

32. F.Yamada, H.Numata, and Y. Taira, "Multi-layered flat-surface micro-optical components directly moled on an LCD panel," Journal of the SID 11(3), 525-531 (2003).

33. T. Hoshino, M. Itoh, and T. Yatagai, "An antireflective grating in the resonance domain for displays," Appl. Opt. 46(5), 648-656 (2007).

34. M. F. Weber, C. A. Stover, L. R. Gilbert, T. J. Nevitt, and A. J. Ouderkirk, "Giant Bire- 
fringent Optics in Multilayer Polymer Mirrors," Science 287(5462), 2451-2456 (2000).

35. M. Piwinski, D. Dziczek, L. Klosowski, R. Srivastava, and S. Chwirot, "Coincidence study of excitation of cadmium atoms by electron impact," J. Phys. B: At. Mol. Opt. Phys. 39, 1945-1953 (2006).

36. R. E. Benenson, "Light polarization by transmission: a laboratory experiment," Eur. J. Phys. 21, 571-578 (2000).

37. N. Stutzmann, H. Jagt1, T. A. Tervoort, C. W. M. Bastiaansen1, and P. Smith, "Novel polarized-light emitting polymer systems: II. use of form birefringent polarizationselective mirrors," Jpn. J. Appl. Phys. 40(10), 5972-5975 (2001).

38. S. Omori, "Polarization and color separator using binary phase grating with subwavelength period," Opt. Rev. 8(4), 254-259 (2001).

39. D. Delbeke, R. Baets, and P. Muys, "Polarization-selective beam splitter based on a highly efficient simple binary diffraction grating," Appl. Opt. 43(33), 6157-6165 (2004).

40. D. Yi, Y. Yan, H. Liu, S. Lu, and G. Jin, "Broadband polarizing beam splitter based on the form birefringence of a subwavelength grating in the quasi-static domain," Opt. Lett. 29(7), 754-756 (2004).

41. X. Deng, J. J. Wang, and F. Liu, "Wideband antireflective polarizers based on integrated diffractive multilayer microstructures," Opt. Lett. 31(3), 344-346 (2006).

42. X. J. Yu and H. S. Kwok, "Optical wire-grid polarizers at oblique angles of incidence," J. Appl. Phys. 93(8), 4407-4412 (2003).

43. L. Zhou and W. Liu, "Broadband polarizing beam splitter with an embedded metal-wire nanograting," Opt. Lett. 30(12), 1434-1436 (2005).

44. M. Born and E. Wolf, Principles of Optics, : Electromagnetic Theory of Propagation, Interference and Diffraction of Light, 5th ed. (Pergamon Press, Oxford ; New York, 1975).

45. A. Tagaya, S. Ishii, K. Yokoyama, E. Higuchi, and Y. Koike, "The Advanced Highly Scattering Optical Transmission Polymer Backlight for Liquid Crystal Displays," Jpn. J. Appl. Phys. Part 1 41(4A), 2241-2248 (2002).

46. B. Paivanranta, N. Heikkila, and M. Kuittinen, "Antireflective subwavelength structured surfaces with enhanced color properties," (2007). To be published at Journal of the Optical Society of America A.

47. C. Huh, K.-S. Lee, E.-J. Kang, and S.-J. Park, "Improved light-output and electrical performance of InGaN-based light-emitting diode by microroughening of the p-GaN surface," J. Appl. Phys. 93(11), 9383-9385 (2003).

48. T.-X. Lee, C.-Y. Lin, S.-H. Ma, and C.-C. Sun, "Analysis of position-dependent light extraction of GaN-based LEDs," Opt. Express 13(11), 4175-4179 (2005).

49. J. B. Cole, S. Banerjee, and M. Haftel, "High accuracy nonstandard finite-difference 
time-domain algorithms for computational electromagnetics: applications to optics and photonics," in Advances in the applications of nonstandard finite difference schemes, R. E. Mickens, ed., pp. 89-189 (World Scientific, Clark Atlanta University, USA, 2006).

50. J. B. Cole and S. Banerjee, "Applications of Nonstandard Finite Differnce Models to Computaional Electromagnetics," Journal of Differnce Equation and Applications 9(12), 1099-1112 (2003).

51. S. Banerjee, J. B. Cole, and T. Yatagai, "Calculation of Dffraction Characteristics of Subwavelength Conducting Gratings Using a High Accuracy Nonstandard Finite-Differnce Time-Domain Method," Opt. Rev. 12(4), 274-280 (2005).

52. J. B. Cole, "High-Accuracy Yee Algorithm Based on Nonstandard Finite Differnces: New Developments and Verfications," IEEE Trans. Antennas Propagat. 50(9), 11851191 (2002).

53. S. Banerjee, T. Yatagai, and J. B. Cole, "Boosting light transmission through interfaces using subwavelength Moth-eye structuring: nonstandard FDTD simulations," 11th Microoptics Conference(MOC'05) H48, 212-213 (2005). 


\section{List of Figure Captions}

Table 1 Comparison of total reflectivities of rectangular grating and triangular grating. The fill factor of the groove is $0.5, \Lambda / \lambda$ is 9.1 and $d / \Lambda$ is 1 . The refractive index of a grating is 1.5 or 3 .

${ }^{a}$ The groove profile is either rectangular or isosceles triangular in shape.

b" Parallel light" is incident light with $\theta i=0$. "Diffusion light" is incident light whose $\theta i$ ranges from 0 to $80^{\circ}$ and whose intensity is uniform.

Table 2 Total reflectivities of experiment and calculation for the surface relief of the grating in case A. The polarizations are TE and TM.

Table $3 d s$ of experiment and calculation for the grating in case A. The periods of gratings are varied from 1.08 to $5 \mu \mathrm{m}$ and the wavelength is $0.6328 \mu \mathrm{m}$.

Table 4 The polarization ratio of TE to TM about total reflectivities of experiment and calculation for the grating with the base and the surface relief in case A. The period is set from 1.08 to $5 \mu \mathrm{m}$ and the wavelength is $0.6328 \mu \mathrm{m}$

Table 5 Angle of the local minimum of total reflectivity in Fig. 22 and $\theta_{B r}$. The refractive index $n$ is 1.5 or $1.9 . d s / \lambda$ is $0.55,0.605,0.64$ or 0.705 . The polarization is TM mode.

Fig. 1 (Color online) Top view and side view of the grating illustrating the method of measuring reflectivity. The dove prism is placed on the back surface of the grating like the tail of a pigeon. Some of the incident light is reflected and detected, and the remainder is transmitted through the grating and is scattered in the dove prism. $\Lambda$ is the period, $d$ is the depth and $d s$ is the substrate thickness of the grating.

Fig. 2 Arrangement of gratings on the mold. The period of the gratings is 1.08, 1.8, 3 and $5 \mu \mathrm{m}$ from left to right. The stripes of the gratings are in the longitudinal direction.

Fig. 3 (Color online) Wavelength dependence of reflectivity on the surface relief in case A. The aspect ratio is varied from 0.5 to $2 . d s$ in Fig. 1 is set to infinity. Regarding the polarization mode, (a) is TE and (b) is TM.

Fig. 4 Grating and incident light. The definition of incidence angle $\theta$ is shown. The side of the grating and its normal line form the angle of incidence.

Fig. 5 (Color online) Total reflectivity of the grating for different aspect ratios and refractive indexes in case A. The incidence angle $\theta$ is defined in Fig. 4. $d s$ in Fig. 1 is set to infinity. Regarding the polarization mode, (a) is TE and (b) is TM.

Fig. 6 (Color online) Total reflectivity of the grating for different aspect ratios and refractive indexes in case A. The incidence angle $\theta$ is defined in Fig. $4 . d s$ in Fig. 1 is set to infinity. Regarding the polarization mode, (a) is TE and (b) is TM. $\Lambda / \lambda$ is 9.1 or 18.2 . The refractive index is 1.5 or 3 .

Fig. 7 (Color online) Total reflectivity of the grating for different aspect ratios and refractive indexes in case B. The incidence angle $\theta$ is defined in Fig. $4 . d s$ in Fig. 1 is set to infinity. 
Regarding the polarization mode, (a) is TE and (b) is TM.

Fig. 8 (Color online) Total reflectivity of the grating for different aspect ratios and refractive indexes in case B. The incidence angle $\theta$ is defined in Fig. 4. $d s$ in Fig. 1 is set to infinity. Regarding the polarization mode, (a) is TE and (b) is TM. $\Lambda / \lambda$ is 9.1 or 18.2. The refractive index is 1.5 or 3 .

Fig. 9 (a) Path of reflected light and the relationship of $\alpha_{1}, \alpha_{2}$ and $\theta_{a}$. (b) Path of reflected light of two times reflection. (c) Path of reflected light of four times reflection.

Fig. 10 (Color online) (a) The axis of electric field. (b)The grating and the field for FDTD calculation (c) The phase distribution of both the incident light and scattered light in TM mode and case A. The electric field along $\mathrm{Y}$ axis is shown. The period is $4 \lambda$ and the aspect ratio is $1 . d s$ is set to zero and a dove prism is removed in Fig. 1. Phase value is relative.

Fig. 11 (Color online) Phase distribution of the scattered light in TE mode and case A. The period is $2 \lambda$ and the aspect ratio is $1 . d s$ is set to zero and the dove prism in Fig. 1 is removed.

Fig. 12 (Color online) Phase distribution of the scattered light in TE mode and case A. The electric field along $\mathrm{Y}$ axis is shown. The period is $3 \lambda$. (a) Aspect ratio is 0.5. (b) Aspect ratio is 1 .

Fig. 13 (Color online) Phase distribution of the scattered light in TE mode and case A. The period is $4 \lambda$ and the aspect ratios of (a), (b), (c) and (d) are 0.5, 1, 1.5 and 3, respectively.

Fig. 14 (Color online) Phase distribution of the scattered light in TE mode for the rectangular grating. The period is $4 \lambda$ and the aspect ratio is $1 . d s$ is set to zero.

Fig. 15 (Color online) Phase distribution of the scattered light in TM mode and case A. The electric field along $\mathrm{Y}$ axis is shown. The aspect ratio is 1 . (a) The period is $2 \lambda$. (b) The period is $4 \lambda$.

Fig. 16 (Color online) Phase distribution of the scattered light in TE mode and case B. The aspect ratio is 1 . (a) The period is $2 \lambda$. (b) The period is $4 \lambda$.

Fig. 17 (Color online) Phase distribution of the scattered light in TE mode and case A. The wavelength is changed and the aspect ratio is 1 . (a), (b), and (c) have periods 3.03, 3.5, and $4.13 \lambda$, respectively. The reflectivities of (a) and (c) are low and that of (b) is high.

Fig. 18 (Color online) Phase distribution of the scattered light in TE mode and case B. The wavelength is changed and aspect ratio is 1 . (a), (b), and (c) have periods 2.84, 3.03, and $3.8 \lambda$, respectively. The reflectivities of (a) and (c) are high and that of (b) is low.

Fig. 19 (Color online) Total reflectivity of the grating against substrate thickness. The polarizations are TE and TM and aspect ratio is 0.5 or 1 .

Fig. 20 Electric field intensity for the gratings with different substrate thickness. (a), (b), and (c) have $d s 0.55,0.65$, and $0.85 \lambda$, respectively. The reflectivities of (a) and (c) are high and that of $(\mathrm{b})$ is low. 
Fig. 21 (Color online) Total reflectivity against $\Lambda / \lambda$ for polarization TE or TM. $\lambda$ was changed. Horizontal axis is log scale. (a) The aspect ratio is 1 , and the refractive index is 1.5 . When $\Lambda / \lambda$ is $9.1, d s / \lambda$ is 0.55 or 0.65 . (b) The grating, substrate 1 and substrate 2 sandwiched by air. (c) The aspect ratio of the grating is 0.5 . The refractive index of substrate 1 and the grating is 1.52 and that of substrate 2 is 1.575 in (b). When $\Lambda / \lambda$ is $9.1, d 1 / \lambda$ is 3.64 and $d 2 / \lambda$ is 181.2 . Fig. 22 (Color online) Total reflectivity of the grating with a base against incidence angle $\theta_{b} . \Lambda / \lambda$ is $9.1, d s / \lambda$ is 0.55 or 0.64 . The polarizations are TE and TM. The transverse axis is the incidence angle $\theta_{b}$. The angle is derived from Snell's law as illustrated in the left figure. (a) The refractive index $n$ is 1.5. (b) $n$ is 1.9. 
Table 1. Comparison of total reflectivities of rectangular grating and triangular grating. The fill factor of the groove is $0.5, \Lambda / \lambda$ is 9.1 and $d / \Lambda$ is 1 . The refractive index of a grating is 1.5 or 3 .

\begin{tabular}{|c|c|c|c|c|c|c|c|c|c|}
\hline \multirow{3}{*}{ Case } & \multirow{3}{*}{ Shape } & \multicolumn{4}{|c|}{ The refractive index is $\mathbf{1 . 5}$. } & \multicolumn{4}{|c|}{ The refractive index is 3.} \\
\hline & & \multicolumn{2}{|c|}{ Parallel light $^{\text {b }}$} & \multicolumn{2}{|c|}{ Diffusion light $^{b}$} & \multicolumn{2}{|c|}{ Parallel light $^{\mathrm{b}}$} & \multicolumn{2}{|c|}{ Diffusion light $^{b}$} \\
\hline & & $\mathrm{TE}(\%)$ & TM(\%) & $T E(\%)$ & TM(\%) & $T E(\%)$ & TM(\%) & $\mathrm{TE}(\%)$ & $\mathrm{TM}(\%)$ \\
\hline \multirow{2}{*}{ A } & Rectangle $^{a}$ & 3 & 3 & 10 & 3 & 24 & 23 & 34 & 14 \\
\hline & Triangle $^{a}$ & 0.18 & 0.01 & 1.67 & 1.30 & 5.0 & 0.2 & 12.7 & 8.3 \\
\hline \multirow{2}{*}{ B } & Rectangle $^{a}$ & 11 & 12 & 45 & 44 & 29 & 27 & 77 & 73 \\
\hline & Triangle $^{a}$ & 6 & 9 & 43 & 45 & 29 & 21 & 66 & 69 \\
\hline
\end{tabular}

${ }^{a}$ The groove profile is either rectangular or isosceles triangular in shape. "Parallel light" is incident light with $\theta i=0$. "Diffusion light" is incident light whose $\theta i$ ranges from 0 to $80^{\circ}$ and whose intensity is uniform. 
Table 2. Total reflectivities of experiment and calculation for the surface relief of the grating in case A. The polarizations are TE and TM.

\begin{tabular}{|c|c|c|}
\hline Mode & Exp.(\%) & Calc. (\%) \\
\hline TE & 0.6 & 0.6 \\
\hline TM & 0.1 & 0.02 \\
\hline
\end{tabular}


Table 3. $d s$ of experiment and calculation for the grating in case A. The periods of gratings are varied from 1.08 to $5 \mu \mathrm{m}$ and the wavelength is $0.6328 \mu \mathrm{m}$.

\begin{tabular}{|c|l|c|c|c|}
\hline Period $(\mu \mathrm{m})$ & 1.08 & 1.8 & 3 & 5 \\
\hline Exp. $(\mu \mathrm{m})$ & 6.0 & 5.3 & 11.2 & 11.9 \\
\hline Calc. $(\mu \mathrm{m})$ & 5.95 & 5.25 & 11.20 & 12.00 \\
\hline
\end{tabular}


Table 4. The polarization ratio of TE to TM about total reflectivities of experiment and calculation for the grating with the base and the surface relief in case A. The period is set from 1.08 to $5 \mu \mathrm{m}$ and the wavelength is 0.6328 $\mu m$.

\begin{tabular}{|c|c|c|c|c|}
\hline$\Lambda / \lambda$ & 1.7 & 2.8 & 4.7 & 7.9 \\
\hline Exp. & 2.2 & 1.7 & 2.1 & 2.2 \\
\hline Calc. & 1.8 & 1.4 & 2.0 & 2.1 \\
\hline
\end{tabular}


Table 5. Angle of the local minimum of total reflectivity in Fig. 22 and $\theta_{B r}$. The refractive index $n$ is 1.5 or $1.9 . d s / \lambda$ is $0.55,0.605,0.64$ or 0.705 . The polarization is TM mode.

\begin{tabular}{|c|c|c|c|c|c|}
\hline$n$ & $\begin{array}{c}\text { TM- } \\
0.55\end{array}$ & $\begin{array}{c}\text { TM- } \\
0.605\end{array}$ & $\begin{array}{c}\text { TM- } \\
0.64\end{array}$ & $\begin{array}{c}\text { TM- } \\
0.705\end{array}$ & $\theta$ Br \\
\hline 1.5 & $30^{\circ}$ & $30^{\circ}$ & $30^{\circ}$ & $30^{\circ}$ & $33.69^{\circ}$ \\
\hline 1.9 & $27^{\circ}$ & $27^{\circ}$ & $27^{\circ}$ & $26^{\circ}$ & $27.76^{\circ}$ \\
\hline
\end{tabular}




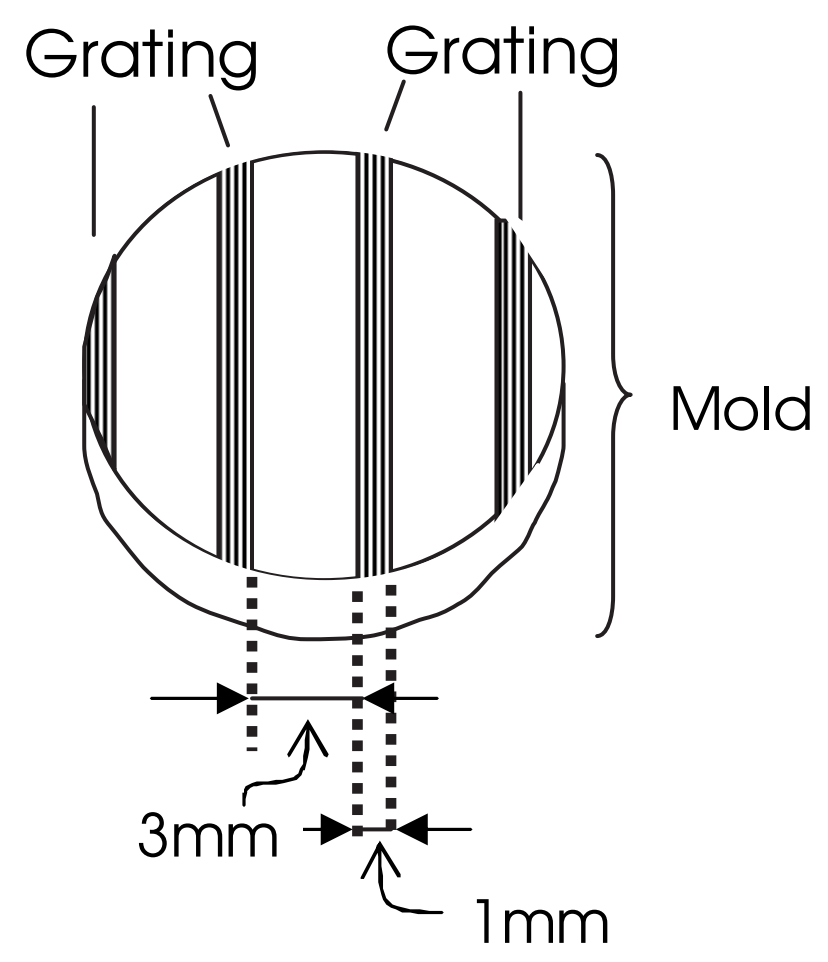

Fig. 1. (Color online) Top view and side view of the grating illustrating the method of measuring reflectivity. The dove prism is placed on the back surface of the grating like the tail of a pigeon. Some of the incident light is reflected and detected, and the remainder is transmitted through the grating and is scattered in the dove prism. $\Lambda$ is the period, $d$ is the depth and $d s$ is the substrate thickness of the grating. 


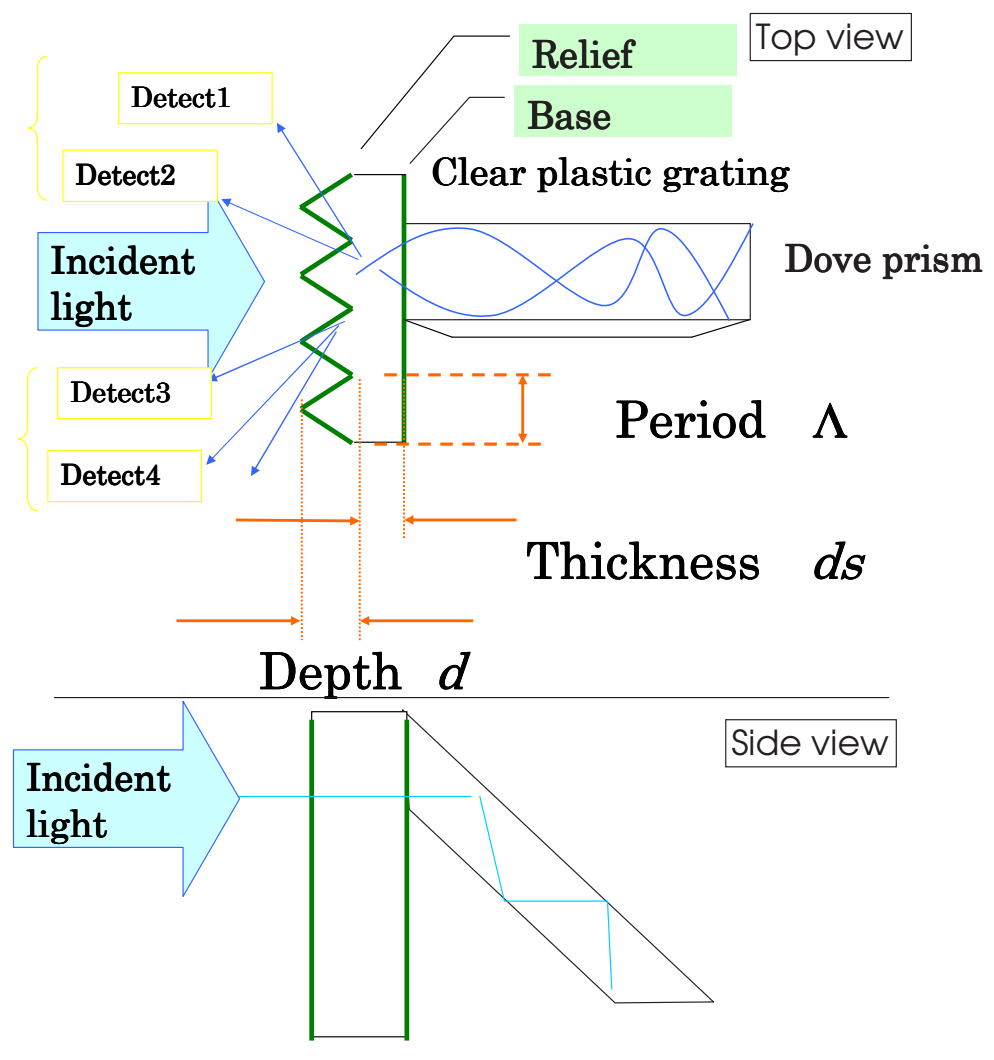

Fig. 2. Arrangement of gratings on the mold. The period of the gratings is $1.08,1.8,3$ and $5 \mu \mathrm{m}$ from left to right. The stripes of the gratings are in the longitudinal direction. 


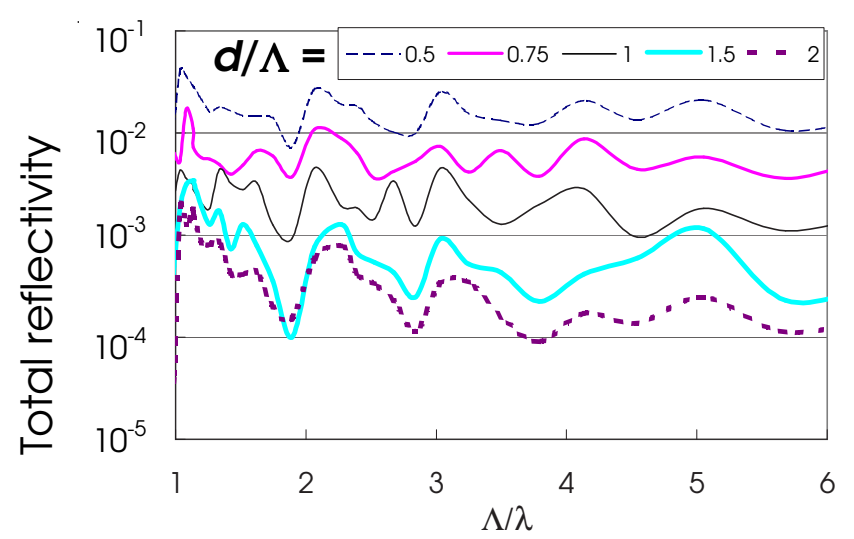

(a) TE mode

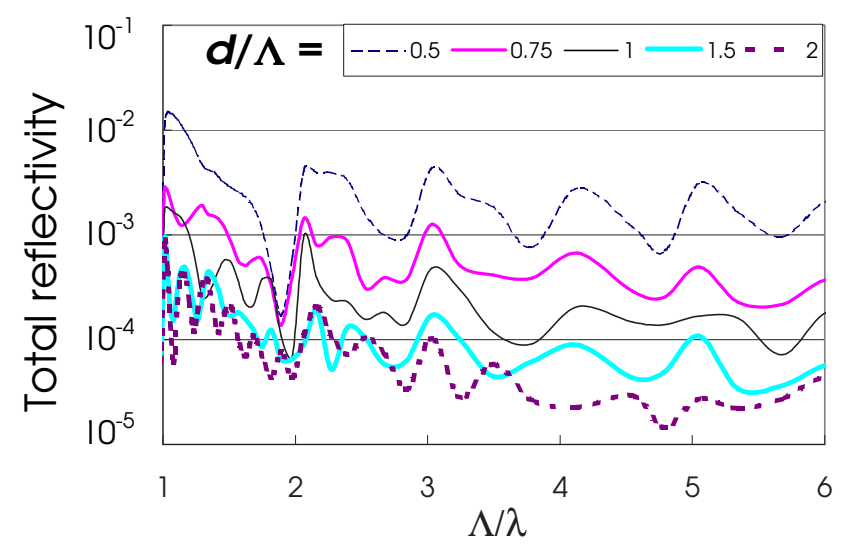

(b) TM mode

Fig. 3. (Color online) Wavelength dependence of reflectivity on the surface relief in case A. The aspect ratio is varied from 0.5 to $2 . d s$ in Fig. 1 is set to infinity. Regarding the polarization mode, (a) is TE and (b) is TM. 


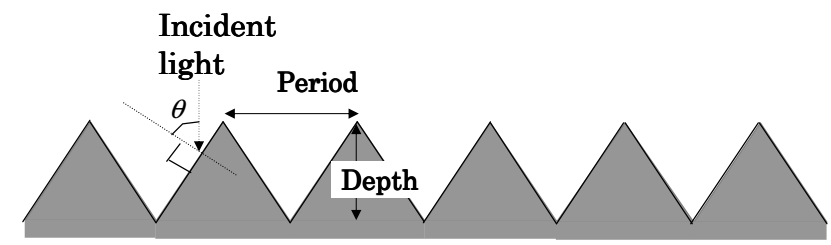

Fig. 4. Grating and incident light. The definition of incidence angle $\theta$ is shown. The side of the grating and its normal line form the angle of incidence. 


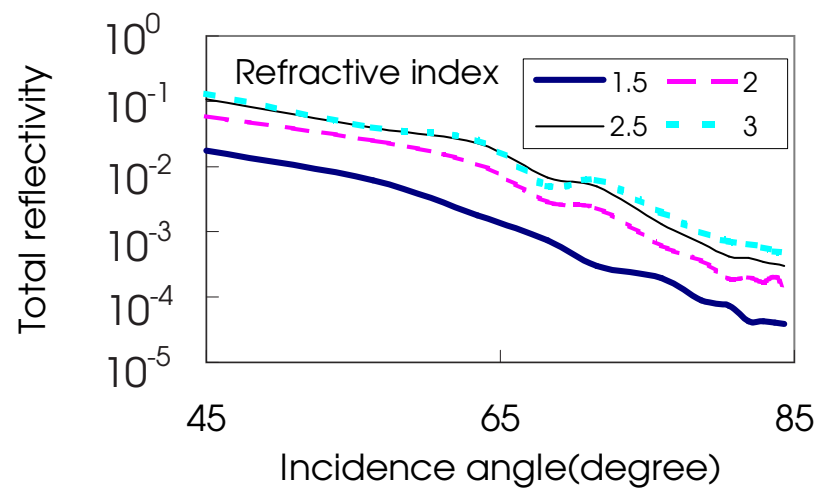

(a) TE mode

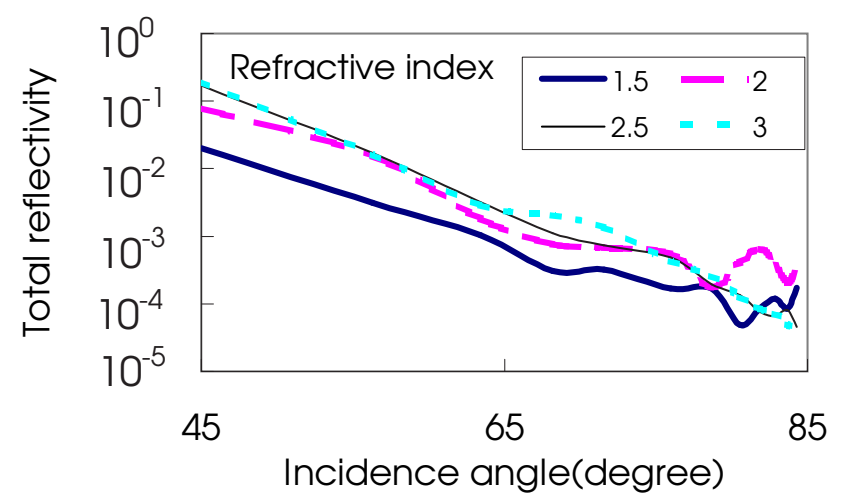

(b) TM mode

Fig. 5. (Color online) Total reflectivity of the grating for different aspect ratios and refractive indexes in case A. The incidence angle $\theta$ is defined in Fig. 4. $d s$ in Fig. 1 is set to infinity. Regarding the polarization mode, (a) is TE and (b) is TM. 


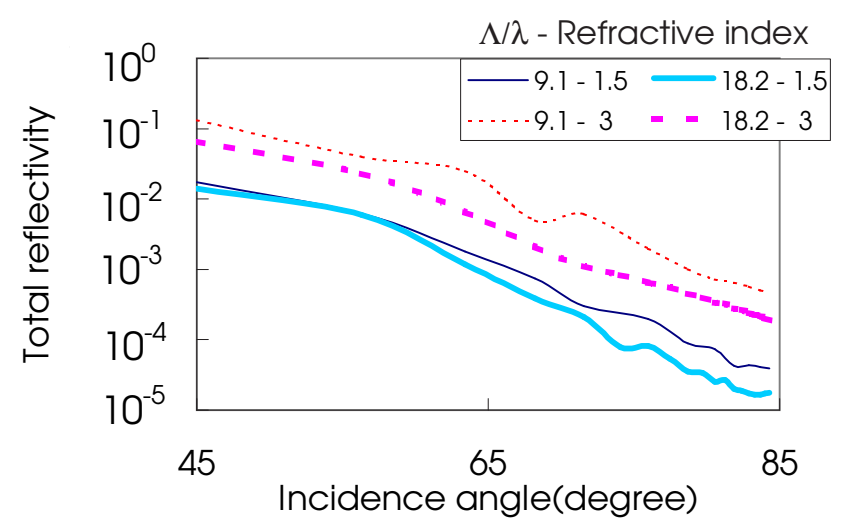

(a) TE mode

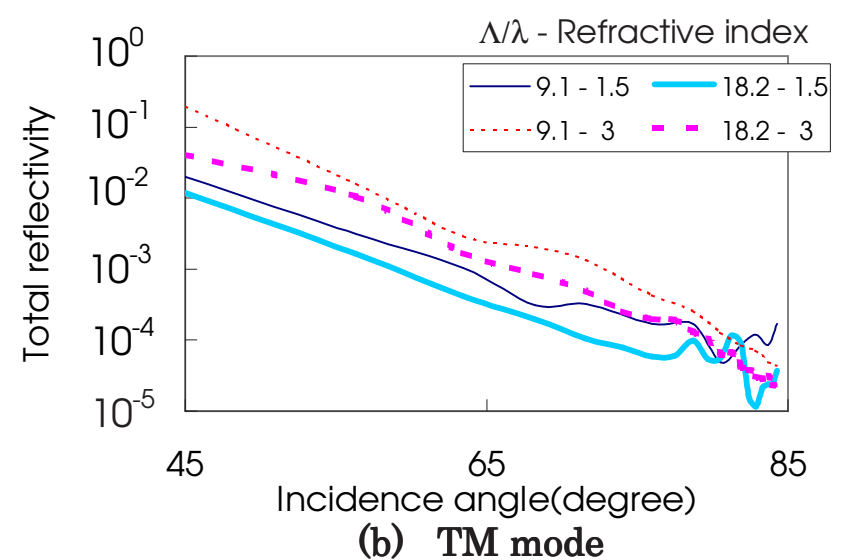

Fig. 6. (Color online) Total reflectivity of the grating for different aspect ratios and refractive indexes in case A. The incidence angle $\theta$ is defined in Fig. 4. $d s$ in Fig. 1 is set to infinity. Regarding the polarization mode, (a) is TE and (b) is TM. $\Lambda / \lambda$ is 9.1 or 18.2 . The refractive index is 1.5 or 3 . 


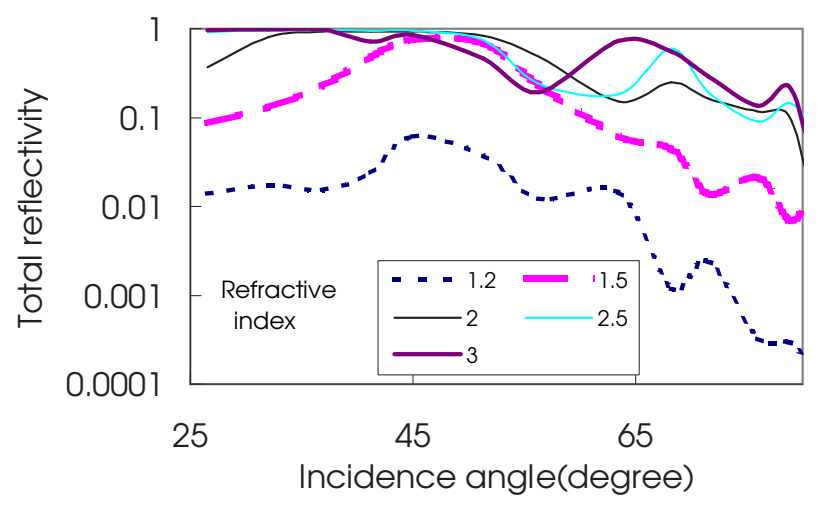

(a) TE mode

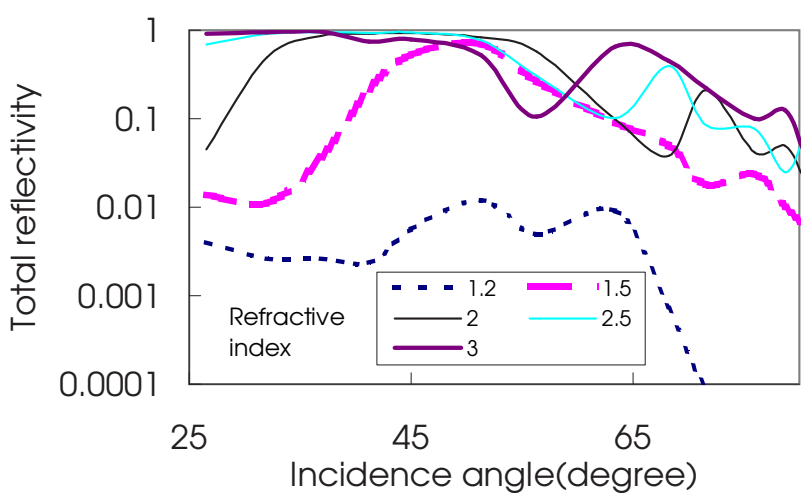

(b) TM mode

Fig. 7. (Color online) Total reflectivity of the grating for different aspect ratios and refractive indexes in case B. The incidence angle $\theta$ is defined in Fig. 4. $d s$ in Fig. 1 is set to infinity. Regarding the polarization mode, (a) is TE and (b) is TM. 


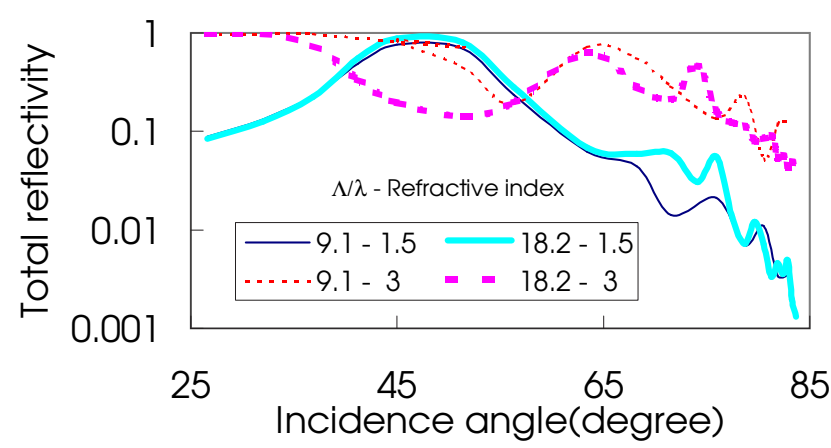

(a) TE mode

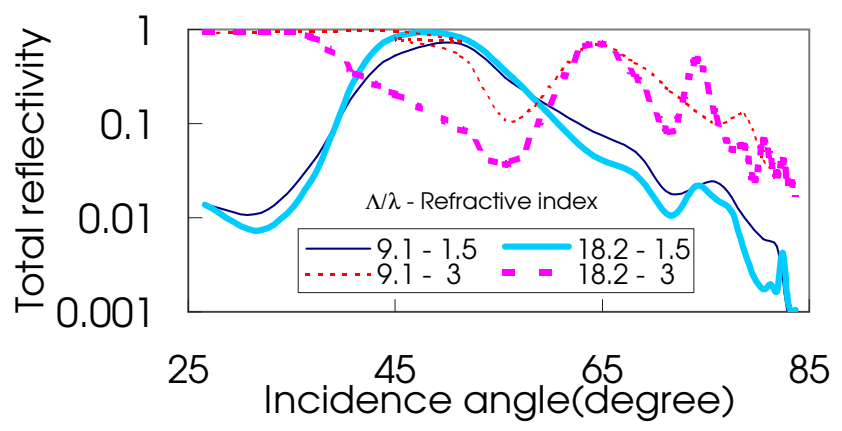

(b) TM mode

Fig. 8. (Color online) Total reflectivity of the grating for different aspect ratios and refractive indexes in case B. The incidence angle $\theta$ is defined in Fig. 4. $d s$ in Fig. 1 is set to infinity. Regarding the polarization mode, (a) is TE and (b) is TM. $\Lambda / \lambda$ is 9.1 or 18.2 . The refractive index is 1.5 or 3 . 


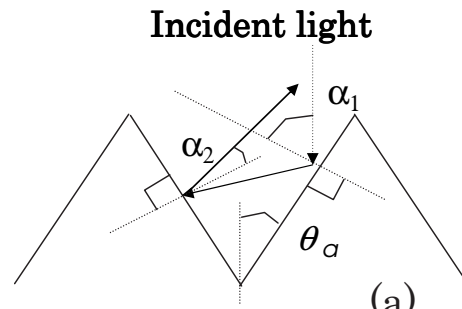

(a)

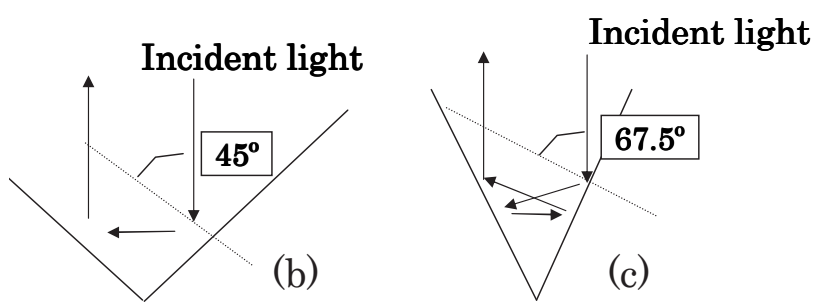

Fig. 9. (a) Path of reflected light and the relationship of $\alpha_{1}, \alpha_{2}$ and $\theta_{a}$. (b) Path of reflected light of two times reflection. (c) Path of reflected light of four times reflection. 


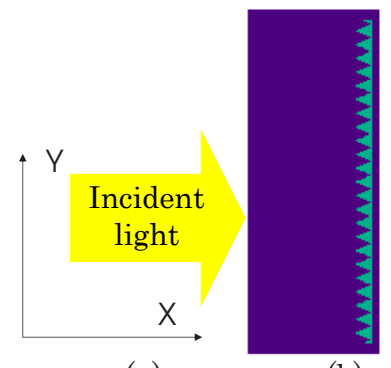

(a)

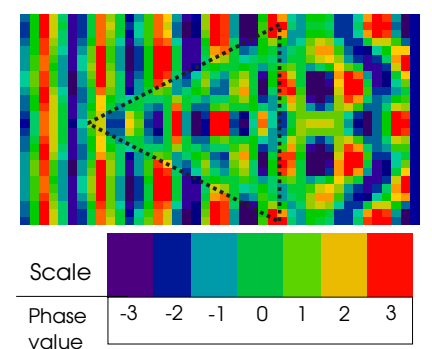

(c)

Fig. 10. (Color online) (a) The axis of electric field. (b)The grating and the field for FDTD calculation (c) The phase distribution of both the incident light and scattered light in TM mode and case A. The electric field along Y axis is shown. The period is $4 \lambda$ and the aspect ratio is $1 . d s$ is set to zero and a dove prism is removed in Fig. 1. Phase value is relative. 


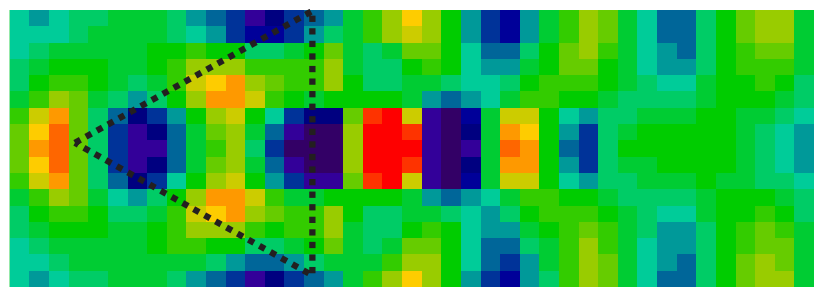

Fig. 11. (Color online) Phase distribution of the scattered light in TE mode and case $\mathrm{A}$. The period is $2 \lambda$ and the aspect ratio is 1 . $d s$ is set to zero and the dove prism in Fig. 1 is removed. 


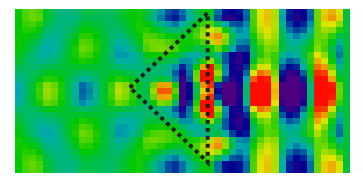

(a) $d / \Lambda=0.5$

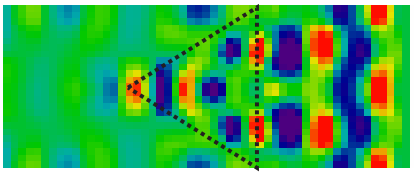

(b) $d / \Lambda=1$

Fig. 12. (Color online) Phase distribution of the scattered light in TE mode and case A. The electric field along $\mathrm{Y}$ axis is shown. The period is $3 \lambda$. (a) Aspect ratio is 0.5 . (b) Aspect ratio is 1 . 


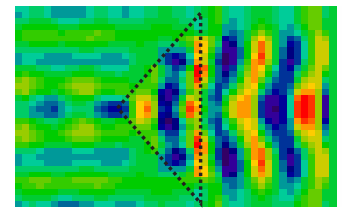

(a) $d / \Lambda=0.5$

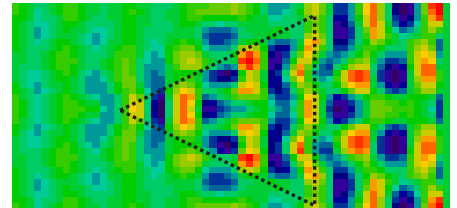

(b) $d / \Lambda=1$

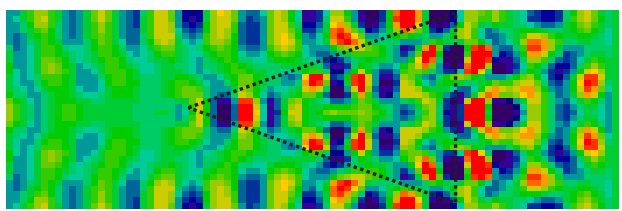

(c) $d / \Lambda=1.5$

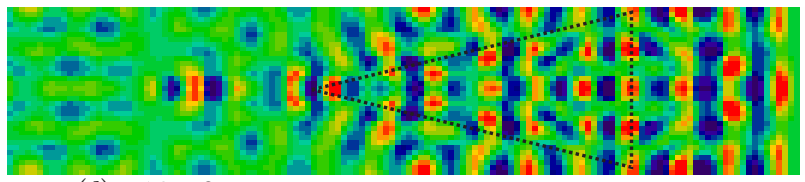

(d) $d / \Lambda=3$

Fig. 13. (Color online) Phase distribution of the scattered light in TE mode and case A. The period is $4 \lambda$ and the aspect ratios of (a), (b), (c) and (d) are $0.5,1,1.5$ and 3 , respectively. 


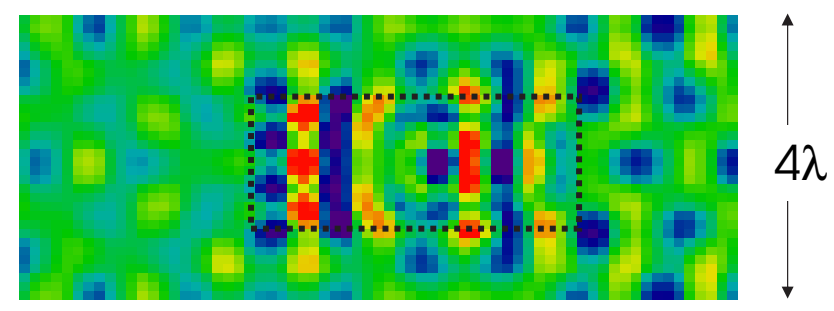

Fig. 14. (Color online) Phase distribution of the scattered light in TE mode for the rectangular grating. The period is $4 \lambda$ and the aspect ratio is 1 . $d s$ is set to zero. 


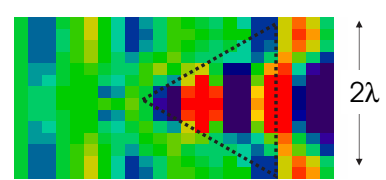

(a)

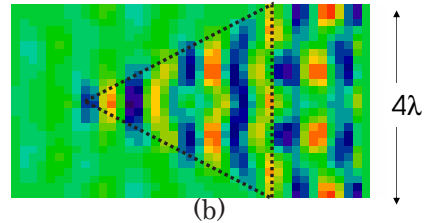

Fig. 15. (Color online) Phase distribution of the scattered light in TM mode and case A. The electric field along $\mathrm{Y}$ axis is shown. The aspect ratio is 1. (a) The period is $2 \lambda$. (b) The period is $4 \lambda$. 


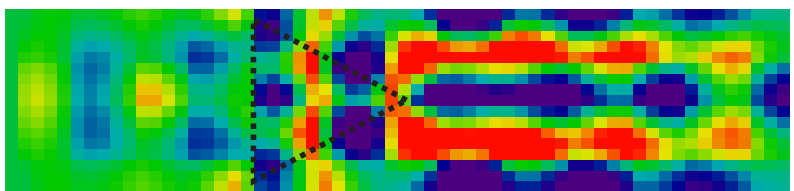

(a) $\Lambda / \lambda=2$

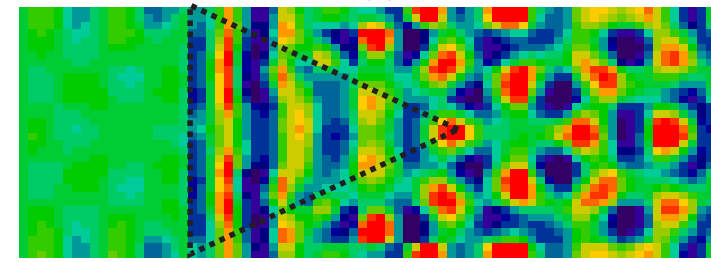

(b) $\Lambda / \lambda=4$

Fig. 16. (Color online) Phase distribution of the scattered light in TE mode and case B. The aspect ratio is 1 . (a) The period is $2 \lambda$. (b) The period is $4 \lambda$. 


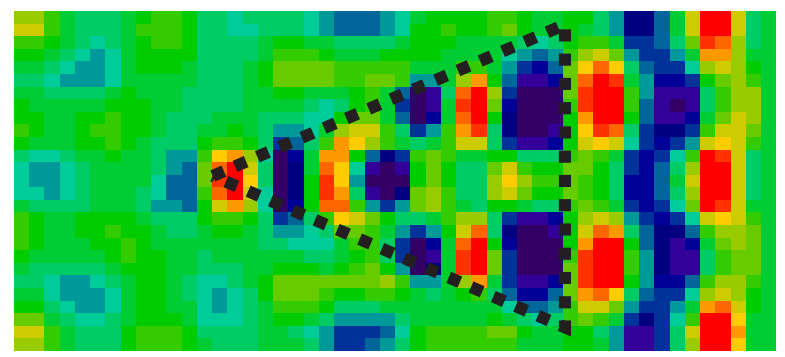

(a) $\Lambda / \lambda=3.03$

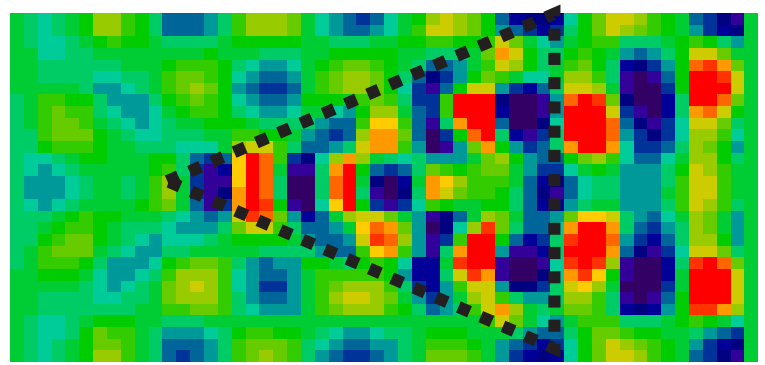

(b) $\Lambda / \lambda=3.5$

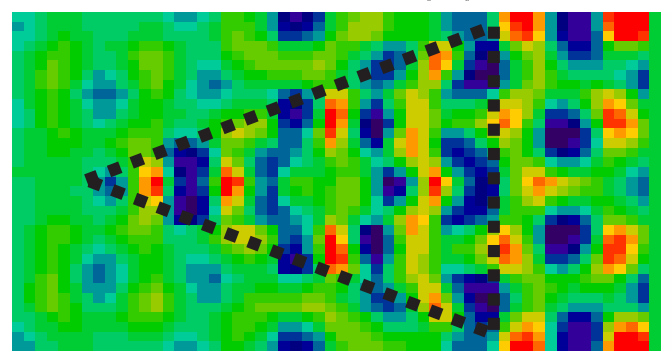

(c) $\Lambda / \lambda=4.13$

Fig. 17. (Color online) Phase distribution of the scattered light in TE mode and case A. The wavelength is changed and the aspect ratio is 1 . (a), (b), and (c) have periods $3.03,3.5$, and $4.13 \lambda$, respectively. The reflectivities of (a) and (c) are low and that of (b) is high. 

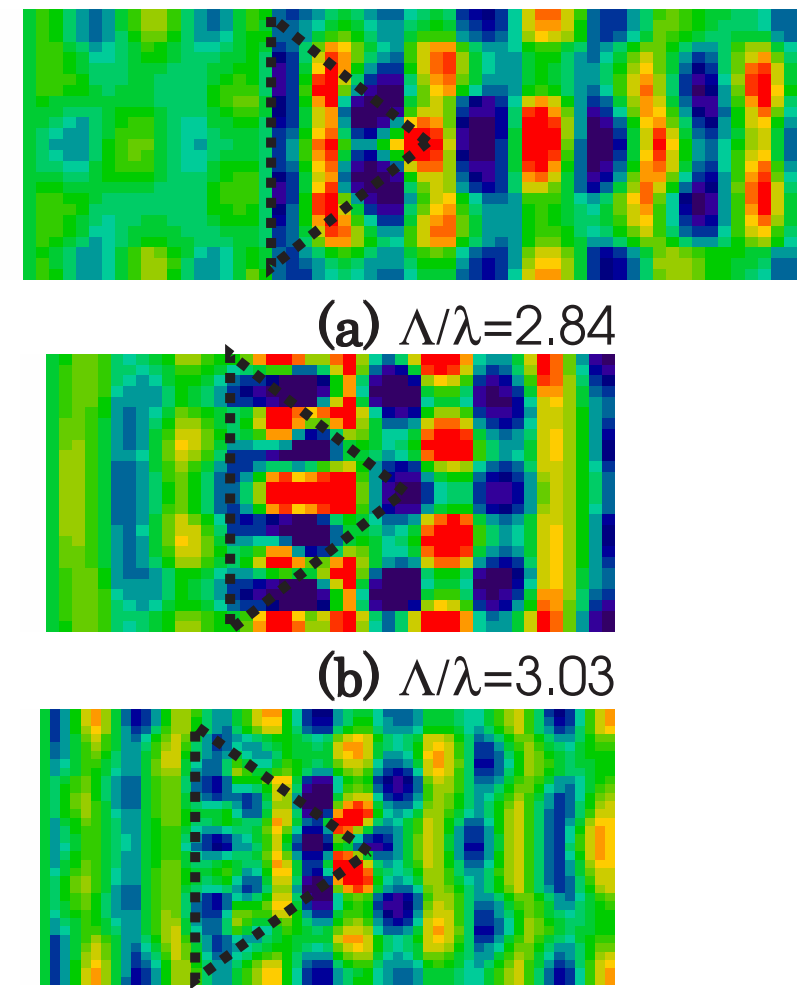

(c) $\Lambda / \lambda=3.8$

Fig. 18. (Color online) Phase distribution of the scattered light in TE mode and case B. The wavelength is changed and aspect ratio is 1 . (a), (b), and (c) have periods 2.84, 3.03, and 3.8 $\lambda$, respectively. The reflectivities of (a) and (c) are high and that of (b) is low. 


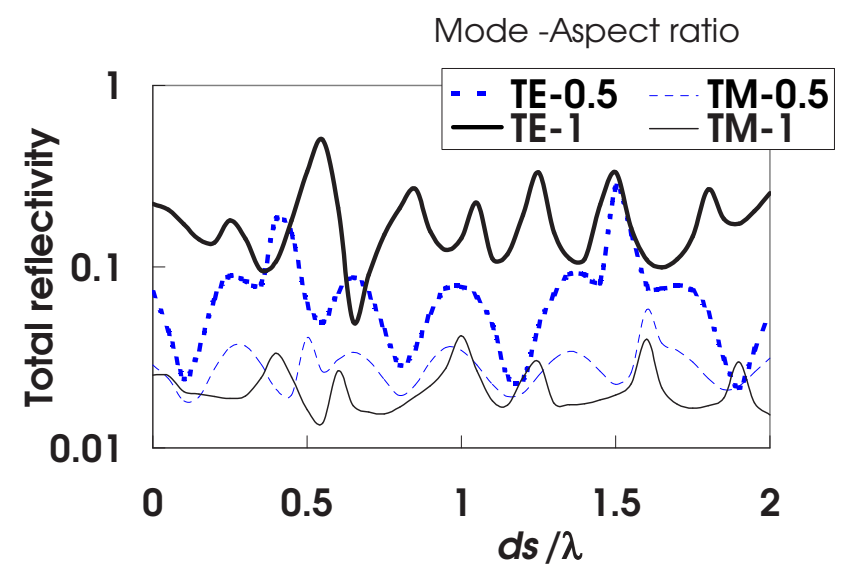

Fig. 19. (Color online) Total reflectivity of the grating against substrate thickness. The polarizations are TE and TM and aspect ratio is 0.5 or 1 . 


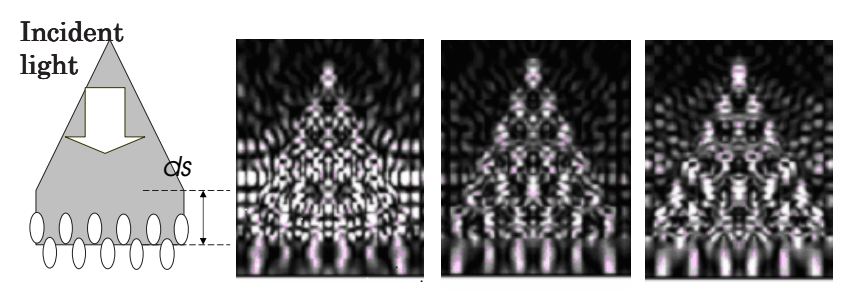

$\begin{array}{lll}\text { (a) } d s=0.55 \lambda & \text { (b) } d s=0.65 \lambda & \text { (c) } d s=0.85 \lambda\end{array}$

Fig. 20. Electric field intensity for the gratings with different substrate thickness. (a), (b), and (c) have $d s 0.55,0.65$, and $0.85 \lambda$, respectively. The reflectivities of (a) and (c) are high and that of (b) is low. 
Mode $-d s / \lambda$

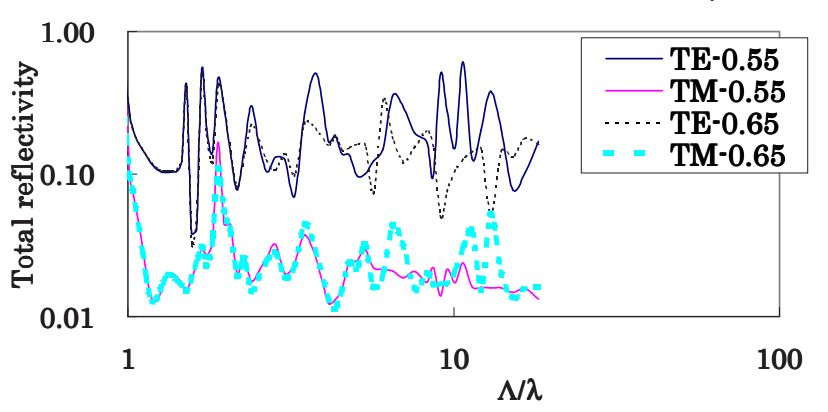

(a)

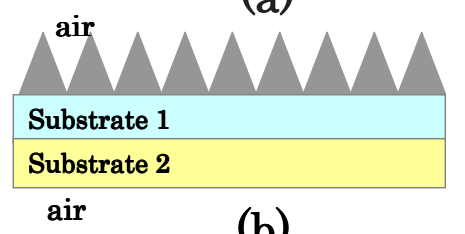

(b)

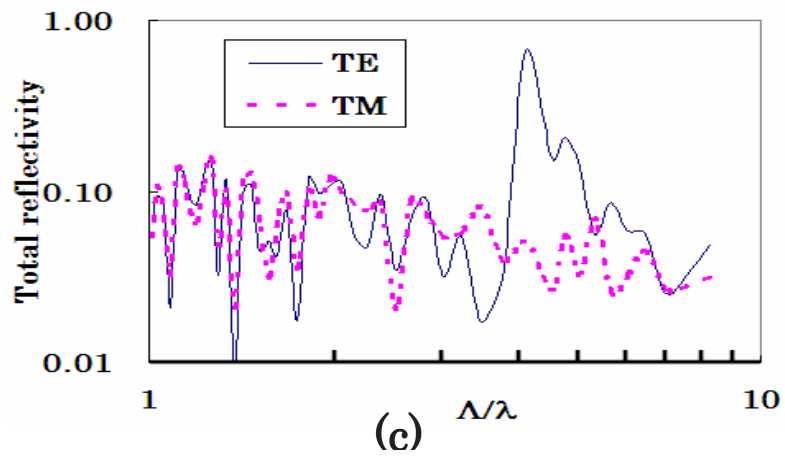

Fig. 21. (Color online) Total reflectivity against $\Lambda / \lambda$ for polarization TE or TM. $\lambda$ was changed. Horizontal axis is $\log$ scale. (a) The aspect ratio is 1 , and the refractive index is 1.5 . When $\Lambda / \lambda$ is $9.1, d s / \lambda$ is 0.55 or 0.65 . (b) The grating, substrate 1 and substrate 2 sandwiched by air. (c) The aspect ratio of the grating is 0.5 . The refractive index of substrate 1 and the grating is 1.52 and that of substrate 2 is 1.575 in (b). When $\Lambda / \lambda$ is $9.1, d 1 / \lambda$ is 3.64 and $d 2 / \lambda$ is 181.2 . 

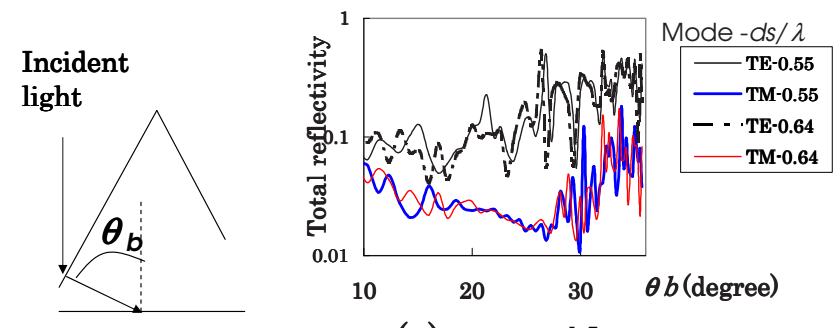

(a)

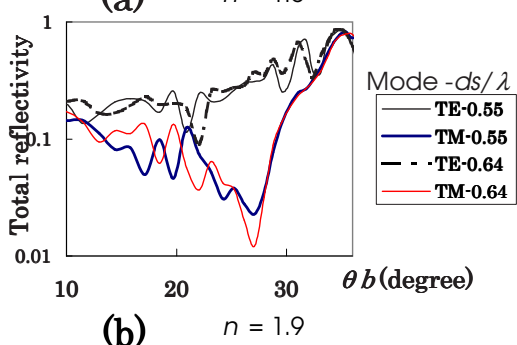

Fig. 22. (Color online) Total reflectivity of the grating with a base against incidence angle $\theta_{b} . \Lambda / \lambda$ is $9.1, d s / \lambda$ is 0.55 or 0.64 . The polarizations are TE and TM. The transverse axis is the incidence angle $\theta_{b}$. The angle is derived from Snell's law as illustrated in the left figure. (a) The refractive index $n$ is 1.5. (b) $n$ is 1.9 . 